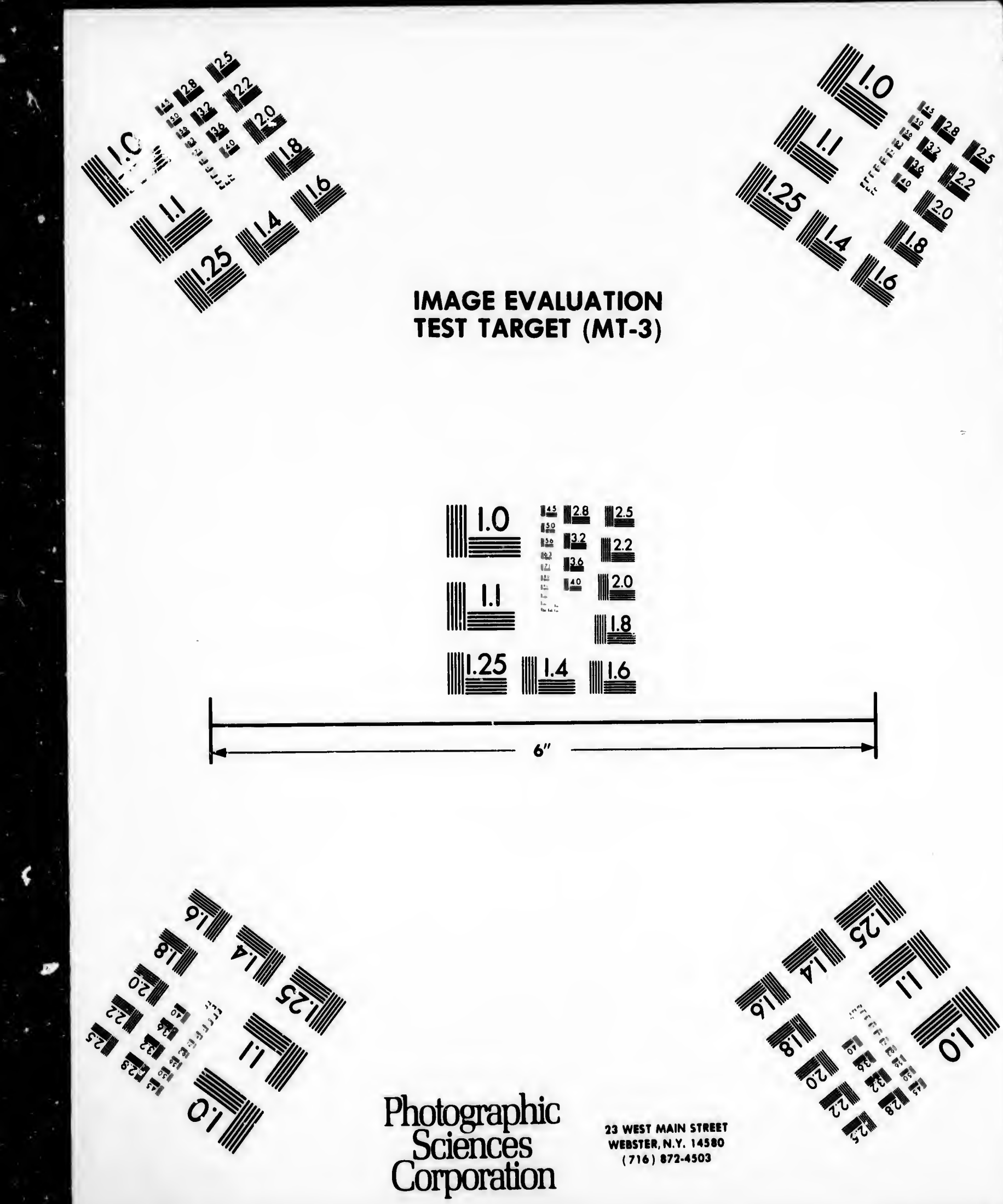




\section{CIHM/ICMH Microfiche Series.}

\section{CIHM/ICMH Collection de microfiches.}


The Institute has attempted to obtein the best original copy avallable for fllming. Foatures of this copy which may be bibliographically unique. which may alter any of the images in the reproduction, or which may significently change the usual method of filming, are checked below.

\section{Coloured covers/}

Couverture de couleur

Covers damaged/

Couverture endommegée

Covers reatored and/or laminated/

Couverture restaurbe et/ou pelliculbe

Cover title missing/

Le titre de couverture manque

Coloured maps/

Cartes géographiques en couleur

Coloured ink (i.e. other than blue or black)/

Encre de couleur (i.e. autre que bleue ou noire)

Coloured plates and/or illustrationa/

Planches et/ou illustrations en couleur

Bound with other material/

Relie avec d'autres documents

Tight binding may cause shadows or diatortion along interior margin/

La reliure serrée peut causer de l'ombre ou de la distortion le long de la marge intérieure

\section{Blank leaves added during restoration may} appear within the text. Whenever possible, these have been omitted from filming/

II se peut que certaines pages blanches ajoutbes lors d'une restauration apparaissent dens le texte. mais, lorsque cela etait possible, ces pages n'ont pas été filmées.

Additional comments:/

Commentaires supplémentaires:
L'Institut a microfilmo le meilleur exemplaire qu'il lui a eté possible de se procurer. Les détails de cet exemplaire qui sont peut-être uniques du point de vue bibliographique, qui peuvent modifier une image reproduite, ou qui peuvent exiger une modification dans la móthode normale de filmage sont indiques ci-dessous.

Coloured pages/

Pages de couleur

Pages damaged/

Pages endommagbes

Pages restored and/or laminated/

Pages restaurbes et/ou pelliculées

Pages discoloured, atained or foxed/

Pages décolorées, tachetées ou piquées

Pages detached/

Pages détachées

$\sqrt{ }$ Showthrough/

Trensparence

Quality of print varies/

Qualité inégale de l'impression

Includes supplementary material/

Comprend du matériel supplémentaire

Only edition available/

Seula edition disponibla

Pages wholly or partially obscured by errata slips, tissues, etc., have been refilmed to ensure the best possible image/ Les pages totalement ou partiallement obscurcies par un fauillet d'errata, une pelure. etc., ont bté filmóes nouveau de façon a obtenir la meilleure image possible.

This item is filmed at the reduction ratio checked below/ Ce document est filmb ou taux de róduction indiqué ci-dessous.

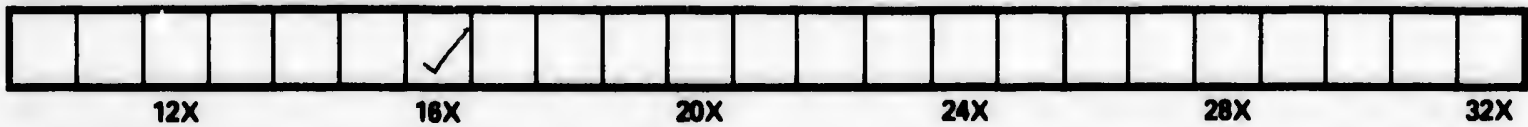


The copy filmed here has been reproduced thanks to the generosity of:

\section{Library of the Public \\ Archives of Canada}

The images appeering here are the best quality possible considering the condition and legibility of the original copy and in keeping with the filming contract specifications.

Original copies in printed paper covers are filmed beginning with the front cover and ending on the last page with a printed or illustrated impression, or the back cover when appropriate. All other original copies are filmed beginning on the flrst page with a printed or illustrated impression, and ending on the last page with a printed or illustrated impression.

The last recorded frame on each microfiche shall contain the symbol $\rightarrow$ imeaning "CONTINUED"), or the symbol $\nabla$ (meaning "END"), whichever applies.

Meps, plates, charts, etc., may be filmed at different reduction ratios. Those too large to be entirely included in one exposure are fllmed beginning in the upper left hand co,ner, left to right and top to bottom, as many frames as required. The following diagrams illuatrate the method:

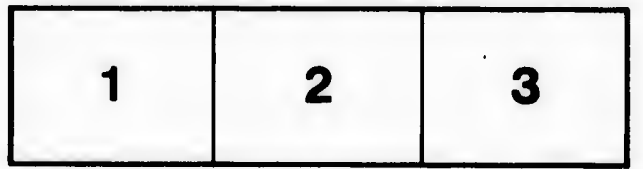

L'exemplaire filmo fut reproduit grace la générosité de:

La bibliothdque des Archives publiques du Canada

Les images suivantes ont bto reproduites avec lo plus grand soin, compte tenu de la condition ot de la netteté de l'exemplaire filmb, et en conformitó avec les conditions du contrat de filmage.

Les exemplaires originaux dont la couverture en papier sst imprimbe sont filmos en commencent par le premier plat ot on terminant soit par la dernibre page qui comporte une empreinte d'impression ou d'illustration, soit par le second plet, selon lo cas. Tous les autres exemplaires originaux sont flimos en commençant par la premiòre page qui comporte une empreinte d'impression ou d'illustration ot en terminant par la dernibre page qui comporte une telle empreinte.

Un des symboles suivents apparaitra sur la derniobre image de chaque microfiche, selon le cas: lo symbole $\rightarrow$ signifio "A SUIVRE", lo symbole $\nabla$ signifie "FIN".

Les cartes, planches, tableaux, otc., peuvent estre filmós des taux de róduction diffórents. Lorsque le document est trop grand pour otre reprodult on un soul clichs, il est filmo a partir de l'angle suporieur gauche, de gauche droite, ot de haut on bas, on prenant le nombre d'images nócessaire. Les diagrammes suivants illustrent la móthode.

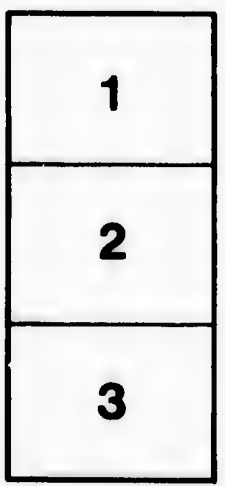

\begin{tabular}{|l|l|l|}
\hline 1 & 2 & 3 \\
\hline 4 & 5 & 6 \\
\hline
\end{tabular}





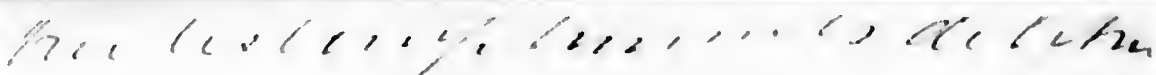

\section{PETIT MANUEI}

\section{I)'AGRICULTURE}

A LUSAGE DES ÉCOLES,

$$
\text { P A B }
$$

Hubert ha Ruve.

APYROLVE PAH IE CONSEHL DE T'INSTRECTJON PCBLIQUE it PAR LA CONSEHL D'AGRHCULTURE.

\section{DEUXIEME ÉDITION.}

\section{QUÉBEC:}

J.A. I, ANGLAIS, I, IBRA IRE, Rut St. Joseph. St. Roch. 


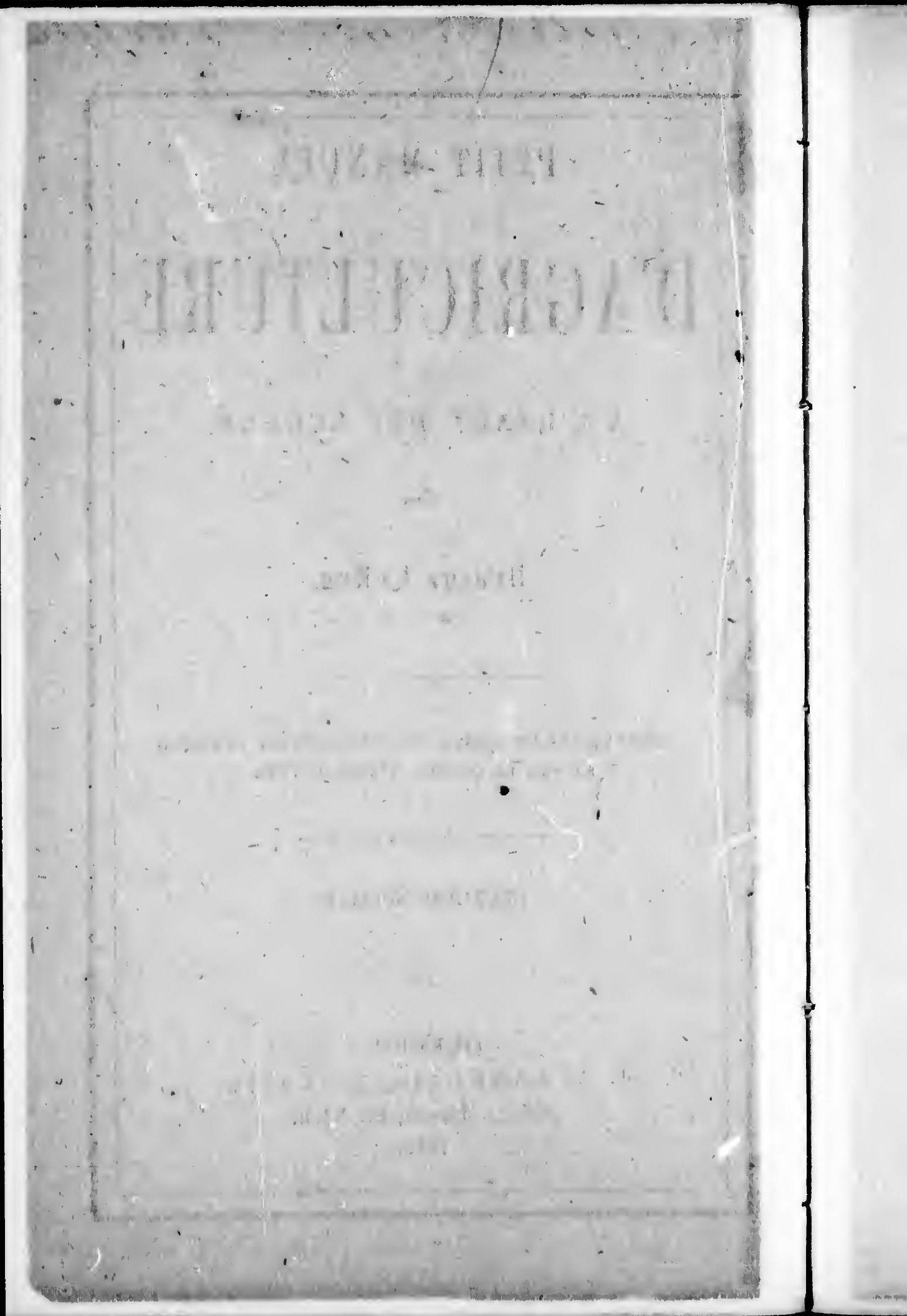




\title{
PETIT MANOEL
}

\section{D'AGRICULTURE}

A LUSAGE DES ÉOOLS,

\author{
PAR \\ HUbert la RUe. \\ APPROUVÉ PAR I.R CONSEIL DL I'INSTRECTION PUBLIQTE \\ et PAR LE CONSEIL. D'AGRICULTURE.
}

DEUXIRXU RDITION.

QUÉBEC:

ATELIER TYPOGRAPHIQUE DE O. DARVEAU,

No. 8, rue Lamrontagne.

1872. 


\section{2 \\ (5)}

Esrearstrí conformément a l'Acte de la Légielaturo Provinciale, en l'année mil huit cent soixante-dix, par Hubert Lu Rus, dans le Bureau du Régistrateur de la Puissance du Canada. 


\section{PRÉ FACE \\ DE LA DEUXIÈME ÉDITION.}

Une premidre édition de einq mille exemplaires du "Petit Manuel " s'est écoulée dans l'espace d'un peu plus d'une année.

Accueilli aveo une bienveillanse toute partiouliere par les journaux do la Province, il n'a pas tardé a recevoir la haute approbation du Conseil de l'Instruction Publique et celle du Conseil d'Agriculture. Plusieurs X instituteurs l'ont introduit dans leurs cicoles arec les résultats les plus satisfaisants, et il est, aujourd'hui, entre les mains de tous les ólèves-maîtres et maîtresses de l'Ecole Normale Lava! de Québec.

Ces témoignages flattcurs disent assez haut que cet opuscule est venu conbler une lacune importante dans l'enscignement de notre jeunesse. Aussi l'autour n'a-til qu'un souhait à exprimer : c'est que l'étude de l'agriculture se propago de plus en plus dans toutes nos 6́coles, dans celles de la campagne sartout.

Cette deuxième édition a êté soigneusemont revue, corrigée, et un peu augmentée. 



\section{P E T I T \\ MANUEL D'AGRICULTURE. \\ Nutrition des plantes.}

Question.-Cumment les plantes se nourrissent-elles?

Réponso.-Les plantes se nourrissent principalement par leurs racines et par leurs feuilles.

Q. Comment agissent ces racines et ces feuilles?

R. Les racines agissent comme autant de petites bouches qui enlevent d la terre une partie de la nourriture dont les plantes ont besoin; et les feuilles trouvent dans l'air l'antre partie de la nourriture qui est nécessaire d la vie de ces mêmes plantes.

Q. Qu'entenil-on par ces mots : terre riche ou fertile, et terre pauvre ou infertile?

R. On dit qu'une terre est riche ou fertile, quand elle renferme, en quantité suffisante, les substances qui sont nécossaires a la nourriture des plantes; on dit qu'une terre est pauvre ou infurtile, quand elle ne renferme pas ces substances, ou ne les renferme qu'en petite quantité.

Q. Pourquoi la plapart de nos terres sont-elles aujourd'hui pauvres ou infertiles ?

R. Parce qu'ayant été cultivées pendant une longue suite d'annces sans fumior, sans engrain, ces terres no contiennent plus les substanoes néoessaires a la uuurriture ot $\mathrm{a}$ la vie des plantes. 


\section{$-6-$ \\ Fopdoes de terres.}

Q. Combien y a-t-il d'especes principales de terres ?

R. Pour les besoins ordinaires do la pratique, on peut diviser les terres en doux especes principales : terres forles ou glaiseusce, terres légères ou sableuses.

Q. Quelle différence y a-t-il dans la composition d'une terre forte et cello d'une terre légère ?

$\mathbf{R}$. Dans les terres fortes, il y a beaucoup de glaise et peu de suble; dans les terres légeres, il y a beaucoup de sable et peu do glaise.

Q. Les terres fortes sont-elles toutes do la même espece?

R. Non; il y a des terres tellement fortos qu'elles sont tout a fait impropres a l'agrioulture. Ises meilleures sont celles qui sont formées d'un mélange de glaise et de sable, en do justes proportions.

Q. Quel nom donnez-vous aux terres formées d'un mélange de sable et de glaise en de justes proportions ?

R. On leur donne généraloment le nom de terres franches.

Q. Les lerrec legeres sont-elles toutes de la même qualitó ?

R. Non; il y a des terres si légeres, comme les sables des rivages, qu'on ne peut pas les cultiver. Les bonnes terres legeres sont celles qui contiennent une certaine quantite de glaisc.

Q. Quels sont les défauts des terres trop fortes?

R. Jues défauts des terres trop fortes sont les suirants : $1^{\circ}$ ocs terres sont pesantes, difficiles ì labourer et à travailler, et se laissent pénétrer diffioilement par l'air, par la chaleur et par l'eau des orages on des plaios; ' $3^{\circ}$ une fojs imbibees d'eau, ces terres la conservent longtemps, et en grande quantité, ce qui, parfois, fait pourrir les racines; $3^{\circ}$ dans les grandos aécheresses, ces terres so fendillont, se crevassent, et len raoines des plantos, se trouvant par la déohaussés, exposées a l'air ou ótoufíés, périssent. 


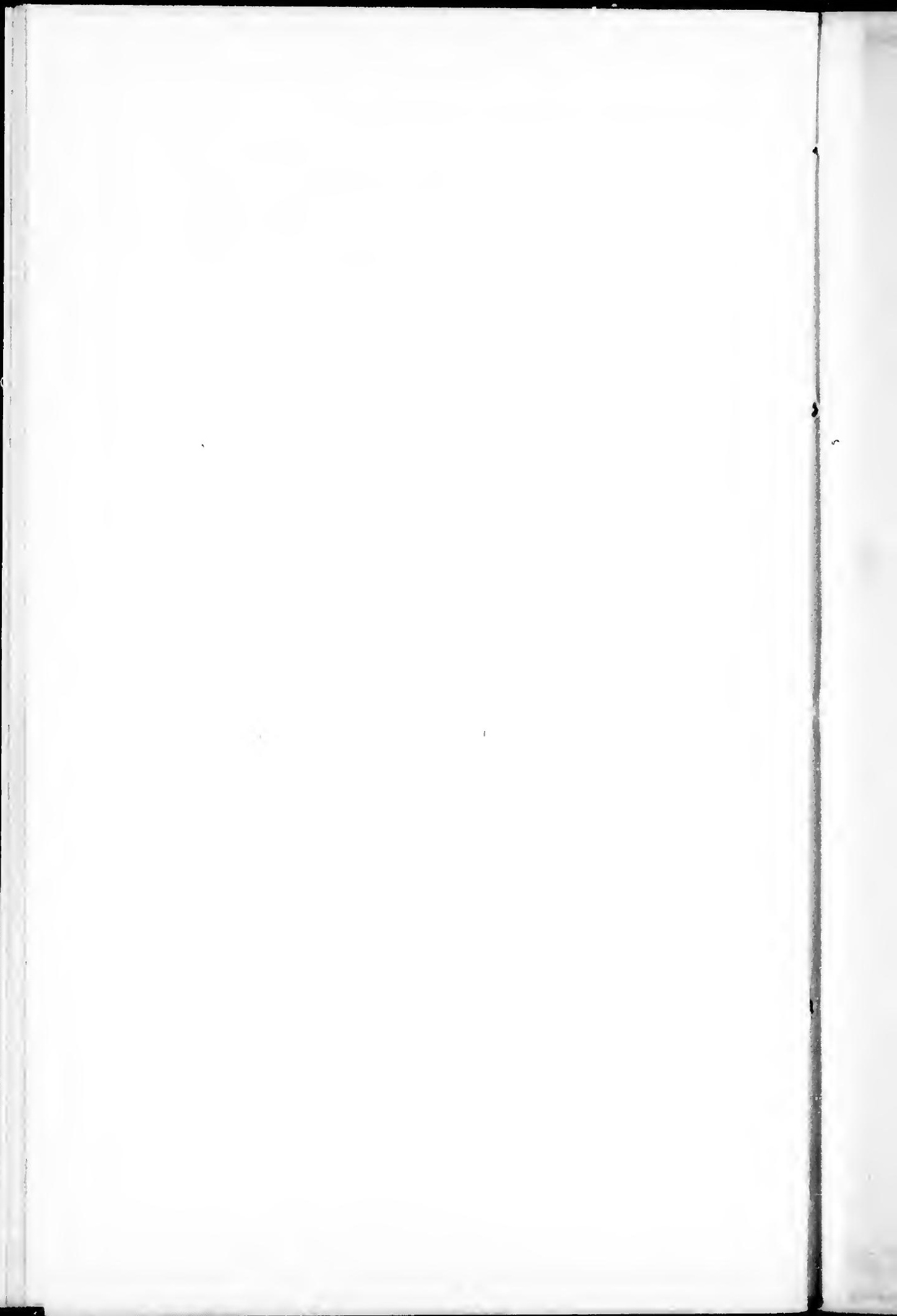

0 
Q. Quels sont les défauts des terres trop légères?

R. Les défauts des terres trop légères sont: $1^{\circ}$ de se laieser pénétrer trop facilement par l'air, et surtont par la chaleur du soleil; $2^{0}$ de ne pas retenir l'eau den orages ou des pluies assez longtemps; et de se dessécher très-vite.

Q. Comment agissent les fumiers äns oes deux especes de terres?

-R. Dans les terres fortes, les famiers se décomposent lentement, et font sentir leurs bons effets pendant longtemps; dans les terres légeres, au contraire, les fumiers se décomposent, rapidement, et leurs bons effets sont de courte durée.

Q. Peut-on diminuer la trop grande force ou la trop grande légèreté des terres?

R. On peut diminuer la trop grande force ou la trop grande légèreté dos terres par des amendements.

\section{Amendements.}

Q. Qu'entend-on par amender une terre?

R. On dit qu'on amende une terre quand ane espesoe de terre on en mêle une autre qui a des qualités differentes, ou enoore; quand on y ajoute certaines substances qui out l'effet de changer ses propriétés.

Q. Donnez des exemples d'amendements.

R. Si je charroie, sur un arpent de terre trop forte, de la terre légdre, du sable, da tuf, des graviers, de la chaux, de la terre de savane, etc., alors je fais un amendement. Si, sur un arpent de terre trop legere, je ohal. roie de la glaise ou de la terre forte, je fais encore un amendement.

Q. Quel est l'effet de ces amendements?

R. L'effet do ces amendements est de rendro les terres fortes plus légères, et les terres légères plus fortes. Ces terres, ainsi amendées, sont meilleures, vu que les engrais de toute espdee, l'eau des pluies et la obaleur, agissent sur elles aveo beaucoup plus d'effioncité 
Q. Ces amendements sont-ils tonjoum faoilen pratiquer?

R. Parfois oes amendements sont faciles a pratiquer; parfois, au contraire, difficiles et oouteux.

Q. Donnez un exemple d'amendements faciles a pratiquer?

R. Si, d uno petito distance d'une terre trop forte, il se trouve une butte de terre sableuse ou légère, de la terre de savane, eto., l'amendement est facile, paree que les oharrois ne sont pas longs, et qu'ils sont peu couteux. $\mathrm{Si}$, au contraire, cette butte de sable, ou cette terre de savane, se trouvent \& une grande distance, alors l'amendement devient couteux a oause de la distanco des charrois.

Q. Quel temps doit-on ohoisir pour pratiquer ces charrois et ces amendements :

R. On doit choisir le temps des morto-saisons, c'esta-dire, ces époques de l'année ou l'on a le moins d'ouvrage à faire.

Q. Les curures des fossés peurent-elles êtro utilisées pour pratiquer ces amendements?

R. Les curures des fossés devraient toujours être utilisces comme amendements. Lorsque ces curures sont composces de terre forte, on devrait les jeter sur les terres trop légères; lorsque ces curures oonsistent en terre sableuse ou tufeuse, on derrait les jeter sur les terres trop fortes.

Q. Que fait-on, ordinairement, des curures des fossos en ce pays?

R. Les curures sont ordinairement déposées sur les robords des fossés et deviennent nuisibles, paroe qu'elles s'opposent au libre écoulement de l'eau, et l'egouttoment.

Q. Lorsque vous avez transporté de la terre forte sur une terre légère, ou do la terre sablense ou légère sur une terre forte, que devez-vous faire ?

R. On doit faire un labour pour bien mélanger oen deux espetoes de terre ensemble. 
Q. Lorique la torre employée comme amendement est de la terre forte, doit-on faire le labour aussitbt aprde lo oharroi ?

R. Isorsque la terre employée comme amendement est do la terre forte, il vaut beaucoup mieux la laisser sur lo terrain pendant quelques rnois avant de labourer, afin do donner aux mottes lo temps de se pulvériser.

Q. Ises amendements, dans cortains cas, peuvent-ils se pratiquer d'une manière encore plus économique?

R. Oui, cels peut so faire, par exemple, lorsquo sous une coucho de terre trop forle, il so trouve une oouche de terre légère; ou lorsque sous une couche de terre trop légire, il se trouve une couche de terro plus forte.

Q. Comment, alors, opérez-vous l'amendement?

R. On opere alors l'amendement par des labours profonds qui mollangent oes deux especes de terres ensemble.

Q. Connaissez-rous quelque autre methode pour amender les terres trop fortes?

R. On amonde encore les terres trop fortes par des labours ot des hersages fréquents, par l'émploi des engrais verts, et par la culture de certaines plantes, oomme les navets, les carottes, les betteraves, les pommes de torre.

Q. Cumment agiseent les labours ot les hersages frequents, et la culture des légumes ?

R. Les labours ot les hersages fréquents, et la cultare des légumes, agissent en pulvérisant la terre, et en en diminuant la consistance et la force.

\section{Travaux de la terre.}

Q. Quels sont les principauz travaux à faire pour l'ensemencement des graines?

R. Les principaux travaux a faire pour l'ensemencement des graines sont : le labour, lo hersage, le roulage, l'Ggouttement, les fumures.

\section{Labour.}

Q. A quoi servent les labours? 
R. Les labours servent $1^{\circ} \mathrm{d}$ ameublír la terre; $2^{\circ} \mathrm{d}$ dotruiro les mauraises herbes; $3^{\circ} \mathrm{a}$ môler la terro aveo bes amendements et les engrais.

Q. Quelles sont les principales espices de labouns?

R. Les principales espèces de tabours sont : les habours profonds, et les labours superficiels.ou légers; les kabours d'autonne, et les labours de printemps.

Q. Qu'entendez-vous par labours profonds ot par larbours supeeficiels?

R. On dit qu'un labour est profond quand ha oharrue eat enfonose $\mathbf{\alpha} 7$ ou 8 pouces dans la terre; on dit qu'un labour eat léger quand la charrue n'est enfoncés qu'd quatre ou einq pouces.

Q. Pratique-t-on, dans certains cas, des labours encore plus profonds que ceux que vous venez d'indiquer?

R. Oni, dans oertains eas, on pratique des labours de 9 a 10 porroes de pmfondeur. On donne d cotte espoce de labour le nom de défoncement. Lo defoncement se pratique a l'aide de charrues particulitres.

Q. Les labours prifonds conviennent-ils également dans toutes les especess de terres ?

R. Les labours profonds conviennent a toutes les terres qui ne sout pas déjł trop légères.

Q. Quels sont les cffets des labours profonds dans los terres fortes ou franches?

R. Les effets des labours profonds dans les terres fortes ou franches sont d'amoublir et de pulveriser une couche de terre plus épaisse.

Q. Quels sont les principaux effets de oet ameublissement profond ?

R. Les principaux effets de et ameublissement profond sont: $1^{\circ}$ de permettre aux racines des plantes de se frayer plus aisément un ehemin a travers la terre pour y puisor leur nourriture; $2^{\circ}$ de permettre a l'eau des pluies de pénétror plus profondément dans la terre, co qui a l'effet d'empêcher eette ean de s'accumuler autour des raoines dans las annćes pluvieuses. 
Q. Oomment l'ear, longu'clib at on trop grande abondanoe autour des racines, pentalle les endommager?

R. Loraque l'eau est en trop grande abondance sutour des racines, olle les fait pourrir, of dotruit les plantes.

Q. Les labouss proforids, sur les cerre fortes, ontils de bons effets en temps de sécherease?

B. Ies labours profonds, sur 1 . terres forles, ont de bens offots en tomps de sécheresse, paree qu'alors l'eau somonto du fond a la surface, ot vient bumooter los raoines.

Q. Dano quels ass les laboure legene convionnent-ile lo mioux?

R. Ines laboure Uyere con Mennent 10 mbax $1^{\circ}$ dans bo terres legtrea, parce qu'ici on no oberohe pas 1 amenblir des wres doja trop meubles; $2^{\circ}$ dans les oas ou ha couche do bonne terne arable eut minos, et qu'au-deswous il so trouve du tuf, des graviers, oto.

Q. Dane quels ous los labours d'automne convionnent-ily le micux?

R. Les labours - ome conviennent le mioux dens les terres fortes ou franches, dans colies, surtoul, qui ont ets longtempw en friohe ou en prairies.

Q. Quels wont les bons effets de ces laboun d'aw tomne?

R. Les bons offets de ces labours d'aulomne sont que les tranches do tarre soulevées par la obarrue, se trourant exponses a l'air et a la gelée, deviennent friables et s'ameublisent; en outre, ces labours d'automne donnent une graade avance pour les travaux du printempe : $\infty$ qui eat d'une grande importanco aveo nos courtes saisons.

Q. Dans quels eas los labours d'automne ne conviennent-ils pase?

R. Les labours d'automine ne conviennent pas dans les terres trop logdres ot trop meubles, parce que l'action de l'air et de la gelée les ameublirait davantago. 
Q. Ies labours d'automns ont-ils encore d'autres inconvenients dans les terres légetres?

R. Oni; dans les terres legeres qui sont en pente, les fortem pluies de l'automne et da printemps emportent uu loin la glaise qui est plus légère que lo sable, et entralnent cette glaise dans les ruisseaux et dans les foraer.

Q. Quel nom donne-t-on généralement is oet effet produit par les grosses pluies sur les labours d'antomne?

R. On dit généralement, alors, que la terre se degraieve. fuit?

Q. $A$ quels signes reconnait-on qu'un labour est bien

R. On reconnaît qu' un labour est -bien fait aux signes mivants: $1^{\circ}$ les tranches de terre coupées par la charrue sont d'égale épaisseur et largeur; $2^{\circ}$ ces tranches sont toutes renversées régulièrement les unes nor les autres, do manièro qu'elles se supportent égaloment; 30 les planches sont un peu plus ólevées au unitiou qu'aux bords; $4^{\circ}$ les planohes sont toutes de undme largeur; $5^{\circ}$ les raies qui les séparent ne sont pas tuop profondes et sont droites.

R. Qual inconvénient présente ane tranche de labour qui ne s'appuie pas également sur une autre?

R. Si une tranohe de labonr ne s'appuic pas également sur l'autre, il se produit des enfoncements, des trous, daus lesquels l'eau s'accumule, ce qui a l'effet de faire périr les plantes, par l'excès d'eau qui noie leurs raoines, ou par les gelées.

Q. Quello ost la meillouro langeur a donner aux Janohes?

B. La meilleure largour a donner aux planches est géWraloment do 12 \& 18 pieds, dans les terres fortes, et flun, dans les terres legères; cela, cependant, peut varier lans certains cas.

Q. Quelle est Is mellleure dircotion d douner aus slanches daps les terres fortes? 


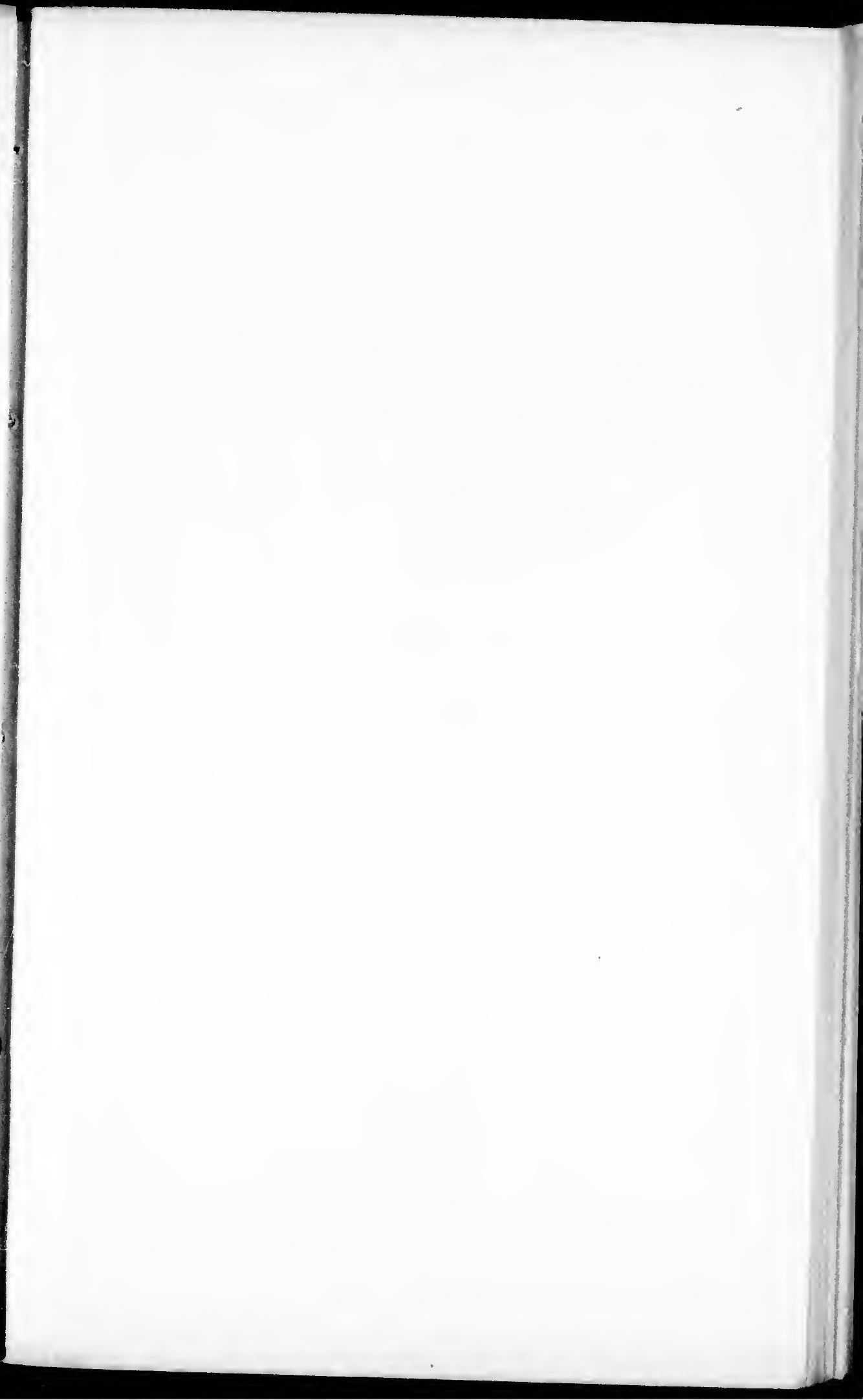




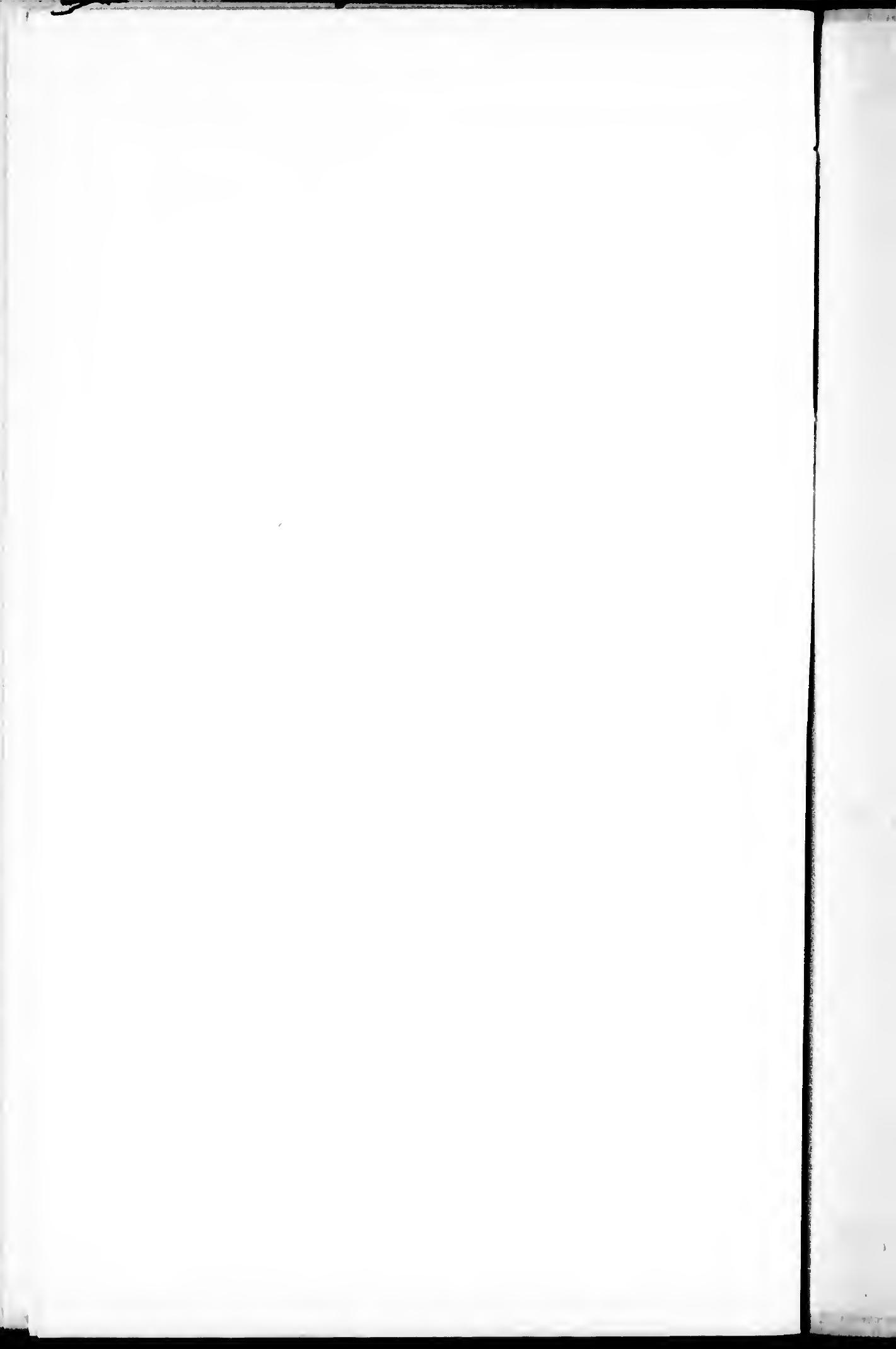


R. La meilleure direction à donner avx planohes, dans les terres furtes, est celie qui facilite lo mieux lour égonttement, o'est-d-dire, la direction qui va suirant la pente naturelle de la pièce.

Q. Pourquni les raies qui séparent les planches doivent-elles être droites ?

R. D'abord, parce que des planehes avec raies droites présentent une plus belle apparenee al l'œil, et enouite, parce que l'egouttement se fait mieux.

Q. Un bon labour a-tril une bien grande influence sur le rendement d'une piece de terre ?

R. Oui; sans un bon labour, il ne peut gadre y sooir de bonnes récoltes ni de bonnes prairiés.

Q. Enumérez les principaux défuuts des labours, tols qu'on les voit en oo pays.

R. Les principaux défauts sont les suivants : $1^{\circ}$ les tranches de terre sont ma! renversées, de sorte que bien souvent, au lieu do s'appuyer les unes sur les autres également, elles sont couchées a plat; $2^{\circ}$ le milieu de la planche, au lieu d'être un peu plus élevé que les bords, est souvent plus bas, ce qui permet a l'eau d'y sojounner et de faine périr les plantes; $3^{0}$ d'autres fois, le mitiou do la planohe est beaucoup trop élevé, de sorte que si cette planche est stroite, elle a la forme d'un pain do sucre $; 4^{\circ}$ les planches sont trop étroites, ce qui augmonte inutilement le nombre des raies et oceasionne une porte de terrain; $4^{\circ}$ les planches sont loin d'awoir toutes la même largøur : ainsi l'on voit, sur la mômo piece, des planches de trois pieds de langour, et d oôté, des planches de dourzo a quinze pieds; $6^{\circ}$ les raies sont généralement en ziz.zag, de sorto que l'eau s'éooule mal, et que la pièo présente a l'cil la plas mauvaiso appenonce.

\section{Hersage.}

Q. Quels sont les effets du hersage ?

R. Los effets du hersage sont $1^{\circ}$. d'ameublir ot de 
pulveriser la terre; $2^{\circ}$ d'enterrer les graines d une profondeur convenable.

Q. Quelles sont les principales variétés de hersece ?

R. On donne aux herses plusieurs formes différentes; les principales variétés sont les suivantes : herses simples, herses doubles, henses $d$ dents do bois, et herres à dente de fer.

Q. Quelle différence y a-t-il entre les herses simples et len herses doubles?

R. Ines herses doubles sont deux fois plus larges que les simples, de sorte que, dans le même temps, on fait, aveo les doubles, deux fois plus d'ourrage.

Q. Le même attelage convient-il aux herses simples et aux horses doubles?

R. Pour les herses simples un seul choral suffit; pour les herses doubles il faut deus chevaux.

Q. Les herses d dents de bois et les herses a dents de fer conviennent-elles également à tous les terrains?

R. Les herses à dents de bois suffisent pour les terres légères ou sableuses; mais pour les terres fortes, les herses $d$ dents de fer sont indispensables, paree qu'elles s'enfoncent mieux dans le sol et l'ameublissent d'arantage.

Q. Un seul hersage est-il toujours snffisant ?

R. Un seul hersage suffit ordinairenent cans les terres légores; mais -dans les terres fortex, il on faut souvent deux, quelquefois trois, et même plus.

Q. Quand on fait deax hersdges sur une torre forte, quello est la meilleure méthode d adopter ?

R. La meilleure móthode a adopter ost d'en faire un sur le long, et l'autre, sur le travers.

Q. Quel temps doit-on choisir de préférenoo pour le hersage?

R. On doit choisir le temps ou la terre n'est ni trop humide, ni trop sèche.

Q. Le hersage est-il une opération bien importante?

R. Lo hersage ost une opération des phas importanten. 
Non-seulement il a l'effet d'enterrer les gratnes, mais encore il ameublit la terre, et permet aux tiges do plantes de sortir avec plus de ficilité.

Q. Quels sont les principaux défauts des hersages, tels que pratiqués en co pays?

R. Trìs-souveut ces hersages sont pratiqués sans oul soin. Dans maintes paroisses encore, on ne connait pas l'usage des herses a dents cle fer ; et, le plus sourent, on se contente de pratiquer un seul hersage, la où il en faudrait deux ou trois, La terre, alors, au lieu d'être hersée, n'est qu'égratignée, de sorte qu'on voit encore toutes les tranches du labour. Grand nombre de pieces ne produisent presque rien, à cause d'un défant de hersage suffisant.

\section{Roulage.}

Q. Comment s'exécute le roulage?

R. Le roulage s'exécute en promenant sur la terre, aprids le hersage, des rouleaux de bois ou de for. Ces roulcaux sont tirés par des ehevaux.

Q. Quels sont les effets du roulage sur les terres fortes?

R. Sur les terres fortes, le roulige a l'effet de briser les mottes, de les réduire en poussière, et, par conséquent, d'ameublir la terre.

Q. Quels sont ies effets du roulage sur les terres légères?

R. Sur les terres légères le passage du rouleau a l'effet de tasser la terre, de lui donner plus de consistance et plus de force.

Q. Quel est le meil'eur temps pour pratiquer le roulage sur ics terres fortes?

R. Pour pratiquer le roulage sur les terres fortes, il faut ehoisir le temps où la terre n'est ni trop humide, ni trop durcie par la sécheresse. Lorsque la terre est trop humide, les mottes, au lieu de se réduire en poudre sous le rouleau, ne font que de saplatir; et, en outre, lo rouleau forme alors une croûte qui, plus tard, se 
durcit, et s'oppose \& la sortie des plantes. Loraque la terra est trop seche, les mottes durcies no se brisent pas sous le poids du rouleau.

Q. Le roulage est-il bien utile sur les terres fortes?

R. Le roulage est trds-utile sur les terres fortes; il compldte l'ameublissement commence par le labour et par le hersage.

\section{figouttement.}

\section{Q. Qu'entend-on par égouttement?}

R. On entend par egouttement toutes les opérations qui ont pour objet d'enlever l'exces d'eau ou d'hunidito contenne dans la terre.

Q. Comment se pratique l'égouttement?

R. L'égouttement se pratique de deux manières: $1^{\circ} \mathrm{par}$ los raies, les rigoles et les fossés ouverts ; $2^{\circ}$ par le drainage.

Q. Comment agissent les raies, les rigoles et les foseds?

$\boldsymbol{R}$. Les raies reçoivent l'excès d'eau ou d'humidité qui se trouve sur les planches; les rigoles regoivent l'eau des raies, et la déversent dans les fossés.

Q. A quels signes reconaissez vous qu'une râie est bien faite?

h. On reconnaît qu'une raie est bien faite aux signes suivants : $1^{\circ}$ elle est légèrement et également en pente, de manidre à permettre à l'eau de s'écouler librement vers la rigole ou vers le fossé; $2^{\circ}$ elle est droite, parce que l'eau s'écoule mieux en snivant une ligne droite qu'une ligne courbe; $3^{\circ}$ il n'y a dans cette raie ni buttes, ni mottes de terre, ni pierres, ni morceaux de bois qui s'opposent a l'ćcoulcment facile de l'eau ; $4^{\circ}$ oes raies ne doivent pas être trop creuses, surtout lorsqu'on se propose d'employer les faucheuses ou les moissonneuses.

Q. Est-il bien important que l'égouttement soit bien conditionné ?

R. En vain le labour, le hersage et le roulage seraient sans reproche; si la terre est mal egouttée, elle restera 
infertile; les mauvaises herbes, les grattes, etc., preudront la pluce du bon grain. Sur les prairies mal égouttóes l'vau se prend en glace, qui oongèle les raoines du foin. Enfin, les terres mal égouttées no peuvent être onsemencées que fort tard au printemps.

Q. Mentionnez un des principaux effets d'uu bon egouttement sur une terre forte et froide?

R. Un des principaux effets d'un bon égouttoment sur uno terre forle et froide est de la rendre plus chaude : cela permet non-seulement d'ensemencer plus tôt au printanps, mais encore de récolter plus tôt a l'automne; et en outre, le rendement est bien plus considérable.

Q. A quelle époquo doit-on surtout corriger les défauty des raies et des rigoles?

R. Pour les pidoes ensemencées en grains ou en kígumes, c'est avant ou aussitôt après l'ensomencoment qu'il faut enrriger ces défauts; pour les pmairies, c'est surtout l'automne avant les gelées.

Q. Quel est lo meilleur temps pour eonstater les défauts des raies et des rigoles?

R. Le meilleur temps est apres les grosses pluies; on voit bien alors les endroits ou l'eau séjourne, ot on comprend mieux les causes qui empêchent l'égouttement.

Q. Quelle est la meihcure forme d donner aux raies, rigoles et fosses?

R. La meilleare forme est celle qui consisto \& leur donner des bords légirement inolinés. Quand les bords sont taillés a pio ils se bouchent constamment.

Q. Quels defauts remarque-t-on dans l'egouttement, tel que pratique dans ce pays?

$\mathrm{R}$. II s'en faut que l'on apporte dans l'égouttement tout le soin désirable. Iue plus sonvent, au lien d'être droites, les raies ot les rigoles sont tortueuses; au lieu d'avoir une pente uniforme et egale, elles sont obstruees par des pierres, des mottes de terre, des morceaux de bois. La terre qui provient des curures des fossés, au 
lieu d'être employêe comme amendement, est jeté de ch "que cóté des fossés où elle forme des abotteaux qui bouchent l'extrénité des planches et des raies, et s'opposent a l'égouttement.

Q. Comment pratiquez-vous le drainxge?

R. On peut pratiquer le drainage de plusieurs manières. Mais, quelle que soit la méthode qu'on emploie, on commence par ouvrir des fossés dont la pente est dans la direction d'un fossé principal plus profond qu'on nomme collecteur.

Q. Que faites-vous ensuite?

12- Ensuite on introduit dans ees fossés, et bout à bout, des tubes de terre cuite qu'on recouvre de terre.

Q. Au lieu de tubes de terre cuite, peut-nn employer: d'antres substances?

R. Au lieu de tubes de terros cuite, on peut employer los pierres, les cailloux dont quelques-unes de nors terres cont garnies.

Q. Peut-on employer le bois pour le drainage, et romment ?

R. On peut employer le bois pour le drainage de la manidre suivante : duns les fossés ouverts conune il a été dit plus haut, on couche deux morecaux de bois rond, de la grosseur du bras ou de la jambe, eomme, par exemiple, des sapins dépouillés de leurs branches. Entre ces deux morceaux de bois on laisse un espace libre de deux a trois pouces. Par-dessus ces deux trongons de sapins, on en couche un troisiène; on mieux on les recourre d'une cronte. On continue ainsi a disposer ces morceaux de bois dans toute la longneur du fossé, jusqu'au fossé principal, ou collecteur. Ces fossés, ou drains, sont ensuite remplis de terre.

Q. Connaissez-vous encoro une méthode du drainage qui peut être facilement employéo en ce pays.

H. Cette méthode consisto dans l'emploi de dosses de pins (slabs) au lieu do pierres ou de trongons d'arbres.

Q. Comment disposez-vous oes dosses? 
R. Absolument comme les tronģns d'arbres; c'esta-dire qu'on couche, au fond des fossés ou drains, deux dosses eôte à côte, en laissant entre les deux un espice de deux à trois ponees. Ces deux dosses sont ensuito recouvertes d'une troisième ou d'une crôtte.

Q. Arrive-t-il sourent que des picees de terre penvent être plus ficeilement et plus éeonomiquement égouttées de cette manise que par des fo isés ouverts?

R. Oui, comme lorsqu'il s'agit d'ígoutter des mares, des terrains bas et marécaceux, ou sourecux, qu'on ne pourrait dessécher 'qu'd l'aide d'un très-grand nombre de fissisés ouverts.

Q. Dites quels sont les avantinges qu'offe lo drainages sur les fossés ouverts dians les terres furtes on franch's?

Q. Les avantages sont les suivints: $1^{\circ}$ un terrain drainé n'étant pas eoupé par des fosoés ouverts, peut être ensemencé dans toute sa longneur et largeur; $2^{\circ}$ l'égoutienent étant beaueoup plus parfiat avee le drain:tge qu'avec les fossés ouverts, il s'ensuit qu'on peut ensemencer beaucoup plus tôt au printemps; $3^{\mathrm{S}} \mathrm{par}$ le drainago les terres fortes et froides devieunent phis chaudes.

Q. Quel est lo résultat de l'expérience des pays étrangers sur lis valeur du drainage?

R. Le résultat de cette expśrienoe est que le drainage seul suffit pour doubler et tripler le rendement de li terre.

Q. A quelle profondeur deirent être oreusées les tranchées dans lesquels on introduit les tuyaux ou autres matérianx de drainago?

R. A la profondeur de trois à quatre pieds, quand le sol est assez profind.

\section{Fngrais et Fumiers.}

Q. Qu'entend-on par engrcis?

R. On entend par engrais toutes les substaneos qui, introduites dans la terre, ont l'offet de l'engraisser, o'est- 
d-dire, de lui fournir les substances dont les plantes ont besoin pour leur nourriture.

Q. Quelles sont les espdces d'engrais qu'on peut lo mieux utiliser en Canada?

R. Ces engrais sont: $1^{\circ}$ lo fumior d'étable; $2^{\circ}$ les engrais verts; $3^{\circ}$ les cendres; $4^{\circ}$ la chaux; $5^{\circ}$ le plâtro; $6^{\circ}$ le poisson et le varech; $7^{\circ}$ les eaux sales do touto espeoo, etc.

\section{Fumier d'étable.}

Q. The cultivateur peut-il aisénont augmenter la quantité et la valeur du fumier d'étable?

R. Jue cultivateur peut aisément augmenter la quan. tité et la valeur du fumier d'étable au moyen des litières.

Q. Qu'entendez-vous par litieres ?

R. On entend par litières toutes les substances qui, étendues sous les animaux ou dans lour voisinage, peuvent leur servir de lit, et se mêler au fumier.

Q. Quelles sont les substances le plus fréquemment omployées comme litieres?

R. Les substances le plus fréquemment employéos comme liticres sont: les pailles de toute espece, les feuilles d'arbres, les mauvaises herbes provenant des sarclages, les fougeres, la sciure do bois, lo tan, la terre de savane, la terre ordinaire, ctc., etc.

Q. Quelles sont les litidres les plus économiques que l'on peut omployer en Canada?

R. A cause de la longueur de nos hivers et do la rareté du fourrage, les litières los plus économiques sont la terre de savarie, et la terre ordinaire.

Q. En quel état doivent êtro emplojéos ces deux espèces de terres? sible.

R. Elles doivent être employées aussi scches que pos-

Q. Comment s'emploient les litières?

R. Quelques litières, commo les pailles, les mauraises herbes, les fougdres, eto., sont étendues sous los animaux, 
et leur servent de lit. D'sutres, comme ls terre ordinaire, la terre de sarane, et toutes les substances terreuses, doivent être étendues dans l'allée qui se trouve en arriero des animaux. Ces dernieres litidres n'ont d'autre objet que d'absorber la partie liquide du fumier, et d'an augmenter la quantité.

Q. Comment agissent les litières?

R. Les litières agissent de plusieurs manieres.

10 les litières pailleuses, employées sons les animaur en assez grande quantité contribuent à les tenir dans un grand état de proprete; $2^{\circ}$ les litières pailleuses, de même que la terre ordinaire, ou les substances terreuses, retiennent les urines ot la partic liquide des fumiers qu'on appelle purin; $3^{\circ}$ elles augmentent la quantitó des engrais et leur qualité.

Q. Les urines des animaux et la partic liquide des fumiers ont-elles une grande valour?

R. Les urines et la partie liquide des fumiers, ou purin, ont beaucoup phus de valeur que la partio solide, et on ne doit rien négliger pour les reoueillir et les oonserver.

Q. $\Lambda$ part l'emploi des litidres, connaissez-vous quelque autre moyen recommandé pour recueillir ces parties liquides ?

R. A part Jemploi des litieres, on reconmande les fosses à purin.

Q. Qu'entendez-vous par fosses d purin?

R. Les fosses a purin sont des réservoirs en magonneries dans lesquelles s'écoulent et tombent, au moyen de dalles placées dans le pavé dey étables, les urines et lo purin. Au lieu de ces réservoirs en magonueries; on emploie souvent des tonneg bien étanches.

Q. A quel usage emploic.t-on le purin ainsi recueiti ?

R. A l'aide d'une poinpe qui plonge dans ces réservoirs ou dans ees tonnes, on arrose les tas de fumier placós sous des abris, ou des tas de terre ordinaire, lesquels prennent alors lo nom de composts, Dans eertains 
pays, on a rose los terres ensemencées ou les jardins aveo ces liquiciśs mélangés d'une certaine qnantitó d'eau.

\section{Traitement des fumters.}

\section{Q. Qu'entendez-rous par ces mots: traitement des fumiers?}

R. On entend par ces mots tout ce qui a rapport a leur conservation, a leur charroi et a leur étendage.

Q. Quels sont les meillours moyens a employer pour conserver les fumiers?

R. Ces moyens varient suivant les saisons. Durant l'hiver, il n'y a aucun inconvénient a laisser les engrais dehors, vu que la nuige et la golée ne neüvent pas les endommager. Mais quand surviennent les grosses pluies du printemps et de l'automne, les chaleurs et les averses de lété, il importe de protéger les fumiers contre l'action de ces pluies et des rayons du soleil.

Q. Par quels moyens peut-on rémédier à ces inconvénients?

R. On peut rémédier à ces inconvénients en établissant, en avant ou en arridre des étables et éouries, des abris sous lecquels on jette les fumicrs jusqu'au moment che leur transport; ou bien encore, on établit, sous les établea, dos caves dans lesquelles on jette les fumiers, au mo .n de trappes pratiquées dans les allées.

Q. Fr'quels tenips convient-il de charroyer les fumiers?

R. Lorsque les fumiers doivent être employés sur des pirces de terres qui se trouvent dans lo voisinage des étables, on peut attendre, pour les charroyer, jusqu'au monent où on est prêt à s'en scrvir. Mais si les fumicrs doiveni êtro employés sur des pièces éloignées des étables, il vaut mieux les charroyor d'avance: et le meilleur temps pour ees elhirrois, en Canida, est l'hiver, l'automne et le printemps, sur les chemius de neige.

Q. Quelles précautions fitutil prendro dans oe char. rois? 
R. D'abord, les fumiers doivent être déposćs en gros tas de 25 a 30 voyages au inoins, et ces tas doivent être placcos dans le voisinage des pièces où ils doivent être employés. Aux endroits où ces fumiers doivent être déposés, il faut avoir soin d'établir, d'avance, des plates-formes de terre ordinaire ou de terre de savane, ayant au moins dix d douze ponces d'épaisseur; on jette le fumier sur ces plates-formes de terre ou de terre de savane.

Q. A quoi servent ces plates-formes?

R. Ces plates formes de terre ordinaire ou de terre do savane servent a retenir les liquides qui s'écoulent des fumiers, et qui, sans elles, seraient perdus. Cette terro ello-même devient un excellent engrais.

Q. Si le fumier ne doit être employé qu'à l'automne, quelles précautions doit.on prendre?

R. Si le fumier ne doit être employé qu'd l'automne, il faut le conserver sous des abris; si, a eause de la distance des lieux où il doit être employé, on est obligé do le charroyer d'avanoe, alors il faut avoir soin de lo recouvrir d'une couche de terre ordinaire ou de terro de savane épaisse de cinq d sir pouces, ou de paille.

Q. Ponrquoi employez-rous cetto couverture do terre ou de paille?

R. O'est, d'abord, ponr protéger les fumiers contro l'action des rayons du soleil et contre les pluies; et ensuite, commo ce fumier chauffe pendant plusieurs mois, il s'en échappe des gaz fertilisants que la couverturo do terre retient et empêche de se perdre. Cetto couverturo devient, alle même, un excellent engrais.

Q. Quel autre moyen devez-vous employer pour diminuer la fermentation du fumier?

R. L'nutre moyen consiste at tasser le fumier, en lo battant avec des pelies, ou en lo piótinant.

Q. Corment agit ce tassement du fumier?

1R. Ce tassement agit on empêchant l'air do pénétrer dans le fumier; car moins il entro d'air dans lo fumier, moins il chauffe. 
Q. Quels sont les dépauts les plus remarquables que l'on observe en oe pays, daus le traitement des fumiers?

R. Les défuuts les plins remarquables, dans le traitement des fumiers, sont les suivants: $1^{\circ}$ on n'errploie que peu ou point de litières; on uegrlige d'employer à cet effer les feuilles d'arbres, leg naauvises herbes, les sarclures, les fougères, la terre de savane, la terre ordinaire, qui auraient l'effet de retenir les urines et les parties liquides des fumiers, et en doubleraient la quantité; $2^{\circ}$ lo fumier est jeté avec nógligence devant les étables, et l'on ne se donne même pas la pcine de disposer d'avance des plates-formes de terre pour recovoir les tiss; $3^{\circ}$ l'endroit où l'on jette ces fumiers est sourent creusé d'avince, on consiste en terro légère et sableuse; de sorte que l'eau des pluies, en lavant le fumier, entraine le purin, e'est-2-dire la partio liquide du fumier, à plusieurs pieds sous terre; $4^{\circ}$ los oultivateurs disposent sourcut leurs fumiers bur la neige, par petits tas, de sorte que quand lo temps de l'enfouir est venu, ce fumier est tout lavé, en éponge, et ne vaut guère mieux que de la paille; $5^{\circ}$ entin, on voit sourent les cultivatours disposer de gros tas de fumicrs dans le voisinage des fossés, des ruisseaux, dans lesquels s'écoule tout le purin.

\section{Diverses espèces de fumters.}

Q. Combien y a-t-il d'especes de fumiers?

R. Il y a quatre espéces principales de fumiers: $1^{\circ}$ fumiers longs, certs ou pailleux; $2^{\circ}$ fumiers pourris, on leurre noir ; $3^{\circ}$ fumiers froids ; $4^{\circ}$ fumiers chauds.

Q. Quelles différenes y a-til entre les deuz premières especes de fumiers?

13. Le fumier long, vert ou partlax est le fumier frais qui n'a pas encore chauffé; le fumier pourri on beurre noir est celui qui a vicilli et chauffé.

Q. Lequel de ces deux fumiers est lo meillenr?

2. En rìglo générale, on peut diro que le fumier qui 
a un peu chauffé et commencé a pourrir est le meilleur de tous.

Q. Y a-t-il quelque inconvénient a laisser le fumier chanffer trop longtemps?

R. Oni; car le fumicr qui chanffe trop longtemps perd, par la même, beaucoup de son poids, de son volume, et de sa vileur.

Q. Dans cquels cas enploie-t-on de préference los fumiers ver's ou prillenx?

R. On les emploie de préférence pour la culture des ponmes de terre (patates), pour celle des navets, des carottes, des navets de Suèle (choux de Siam), et : stres lénumes. Dans les terres fortes, les fumiers longs ou paillux ont l'avantage d'ameublir la terre, tout en l'engraissint.

Q. Dins quels cas donne-t-on la préférence mux famiers pourris?

R. On donne la préfírence aux fumiers pourris, quand on veut recouvrir d'engrais une prairic déja faite.

Q. Quels inconvénients présentent les fumiers verts?

R. I. ses fumiers verts présentent l'inconvénient de contenir souvent de mauvaises graines qui, plus tard, germent et infustent les champs en culture.

Q. licmploi des fumiers verts sur les champs qui doivent être ensemencés en légumos présente-il au mêmo decrré èt inenvénient?

R Non; car les sarchages nombreux auxquels ces chimule sont soumis ont l'effet de détruire les mauvaises herbes; mais, même dans ce cas, les fumiers qui ont commiencé a chanffer valent mieux.

Q. ILes matuviises graines se rencontrent-elles aussi dans les fumiers pourris?

R. Jes mavaises graines ne se trouvent pas dans les fumicrs pourris, parce que, pendaut la putréfaction du fumier, ces graines se décomposent.

Q. Qu'entendez-vous par fumiers froids et fumiers chauds? 
R. Les fumiers froirls sont ceux qui chauffent et so pourrissent lentement et difficilement : tes fumiers chauds sont ceux qui chauffent, et pourrissent vite. fumiers.

Q. Donnez des exumples de ces deux espices i?

R. Les fumicrs de vache, de porc, sont des fumicrs froids : les fumicrs de cheval, do mouton, sont des fumiers chunds.

Q. Qeul est le meilleur moyen i employer pour hâter la putréfiction des fumiers troidis?

R. Le meilleur moyen ennsiste a mêler à ces fumiers froids une eertaine quantité de fumiers chauds.

\section{Engrais verts.}

Q. Qu'entend-on par engruis verts?

R. On entend par des mots l'enfouissement dans la terre, a l'aide de la charrue, de plantes encore vertes, yui ne sont pas mûres.

Q. Quelles sout, de toutes les plantes, celles qui convienuent le uieux eomme engrais vert en ce pays?

R. Ise trìfle et le sarrazin.

Q. Comment feriezovous un engrais vert avec le sarrazin?

R. J'enscmencerais lo terrain au commencement de juin. Isorsque le sarrazin aurait atteint sa hauteur, c'est-i-dire, lorsqu'il serait en fleurs, je passerais le rouleau sur la piece pour abattre les ticos. Ensuite je labourerais la piece et enfoinirais le sarrazin. Quand le sarrazin doit être ainsi employé comme engrais verl, il doit être semé très-fort.

Q. Comment feriez-vous un engrais vert avec le trèfle?

R. De la même manière qu'avec le sarrazin: il doit etre cufoui quand il est en fleurs et avant qu'il soit mûr. sol?

Q. Laquelle de ces deux plantes enrichit mieux le 
R. C'est le trefle; mais tandis qu'il exige une terre déjd assez riche pour lui permettre de pousser, le sarrazin, au enntraire, vient bien, même dans les sols pauvres.

Q. Quelle objection les cultivateurs ont-ils ordinairement à l'emploi des engrais verts?

R. Leur plus grande objection est qu'il leur en coate d'enfouir dans la terre une récolte de trèfle ou de sarrazin qui leur rapporterait un certain profit.

Q. Ne croyez-vous pas que eette objection est, parfois, foudée?

R. Oui : si le cultivateur est bien paurre, et si oette récolte est nécessaire pour les besoins pressants de sa famille. Si, au contraire, le cultivateur peut se passer de cette récolte, il trouvera à la fin qu'il y a bénéfice pour lui de l'enfouir.

Q. Comment expliquez-vous le gain par suite de l'enfouissement de ces plantes?

R. A la suite de l'enfouissement de ces plantes, la pièce de terre rapportera deux ou trois fois plus l'année suivante, et pendant plusieurs années à venir.

Q. Les engrcis verts agissent-ils seulement commo engrais?

R. Les engrais verts agissent encore comme amendements; ils ameubiissent les terres fortes, et donnent de la consistance aux terres légères.

\section{Chaux et chaulage.}

Q. Que pensez-vous de la chaux comme engrais ?

R. La chaux est un des principanx engrais d entployer en ee pays; d'abord, pareé qu'elle est nécessaire à la nutrition des plantes, et que la plupart de nos terres en sont dépourvucs; ensuite, parce que les résultats sont surpren:nts, et que le prix en est peu ólevé.

Q. La chaux convient-clle it toutes les especes de terres? 
R. In chanx convient surtout aux terres fortes et nux terres franches, parce qu'elle agit sur elles, nonsouinment oomule engrais, mais aussi comme amendenemt.

Q. N'y a-t-il pas des terres qui contiennent déja trop de chinx?

R. 'Jes terres sont rarcs en ce pays.

Q. Fn quel état cetts chaux doii-clle être employise?

R. On doit l'employer fraîchement éteinte et réduite en poudre tres-fine.

Q. Comment éteignez-vous cette chaux?

R. On peut l'éteindre de trois manières: $1^{\circ}$ en mettant la chitux vive sous un hangar ou sous un abri, et la laissant s'éteindre peu ì peu, à l'aide de l'humiditó de l'air ; $2^{\circ}$ en la mettant sous un abri ou en plein air, et l'éteignant avec de l'cau jéée en petite quantité; $3^{n}$ en la mettant en tas sur la terre, et la recouvrant de terre ordinaire, de terre de savane, de mauvaises herbes ete. : c'est ce qu'on appelle fitire des composts.

Q. Qu'arriverait-il si vous arrosiez la chaux arec uno trop grande quantité d'eau?

R. En arrosant la chaux avec une trop grande quantité d'eau, on courrait le risque de la noyer, c'est-i-dire qu'elle ne s'éteindrait pas ; ou, si cette eau était ajoutée apres que la chaux fût éteinte, cette chaux se prendrait en nasses, et formerait une espèce de mortier.

Q. Comment employez-vous eette chaux ?

R. On peut employer cette chaux de deux manieres différentes; sur la semence, ou sur la terre avant lo labour.

Q. Comment enıloieriez la chaux sur la semence?

R. La meilleure maniere d'employer la chaux sur la semence est de la répándre a la main on à la pelle sur les grains mêmes, aussitôt après qu'ils out été ensemencés, et de herser aussitôt.

Q. Est-il toujours bien facilo de répandre cette chaux?

R. Non; car, pour que cet épandage soit fait d'uno 
cmniere r'gulière et uniforme, il faut que lo temps soit alame, ou qu'il y ait peu de vent. Lorsque le vent est fort, il emporte la chaux au loin.

Q. Quelle est la meilleure méthode à employer pour répandre cette chaus à la main sur la semence?

R. La meilleure méthode est la suivante: on suspend à son cou, ì l'aide d'une courroie, une chaudière remplie de chaux éteinte que l'on prend sur un tas déposé auprès, ou dans une brouette que l'on fait passer dans la raie. La main droite, garnie d'une mitaine de cuir, est armée d'un instrument de fer ou de fer-blane solide, ayant la forme de ceux dont se servent les épiciers pour prendre le sucre dans les boucauts. A l'aide de cet instrument, on prend la chaux dans la chaudière et on l'étend.

Q. D'après quelle autre méthode Ia chaux peut-elle encore être employee?

R. La chaux est employée encore avec suecès de la manière suivante: supposons qu'il s'agisse de relever une vicille prairic couverte de mousse et de mauvaises herbes ; aussitôt avant le passage de la charrue, on répand sur l'herbe la chaux éteinte, qui, l'instant d'après, se trouve enfonic. I'automne suivant, avant le deuxième labour, on fait un nouveaú chaulage, de la même manière. Si cette pièce reçoit un troisième labour, on fait un troisième chaulage.

Q. Que pensez-rous de la chaux employée d'après cette dernière méthode?

R. Je crois que cette dernière méthode offre un des moyens les plus simples et les plus économiques de ramener la fertilité sur bier des terres épuisées en ce pays.

Q. Quels sont les principaux avantages que présente la chaux employée d'après ces deux méthodes?

$R$. Les principaux avantages de la chaux sont: $1^{\circ}$ d'agir comme amendement sur les terres fortes, Jorsqu'elle est employée en assez grande quantité; c'est-ì-dire, qu'elle diminue la consistance et la force de ces terres; $2^{\circ}$ de 
dêtruire les mauvaises herbes, et de faire périr plusieurs iusertes et nnimaux nuisibles; $3^{\circ}$ d'agir comme engrais.

Q. Quelle quantite de chaux doit-on employer, en suivant l'une ou l'autre de ces denx méthodes?

Q. En suivant la première méthode, la quantité de chaux it entoloyer virie de 30 it 50 minots par arpent. En suivant la deuxieme míthole, il en faut employer de 30 is 50 minots sur ehmua labour.

Q. Ce moyen d'améliorer la terre est-il dispendieux?

R. Non; la ehaux coûtant, rendue sur les lieux, cinq ou six chelins la barrique de 6 minots; supuo ant qu'on répande 36 minots sur un arpent,, le eoût de cette challx n'est que de trente ou trente.six chelins par arpent.

Q. Quels résultats pourcz-vous attendre de la chaux employíe d'aprìs ecs deux méthodes?

R. En elluployant la chaux d'après ees denx methodes, on peut s'attendre il obtenir des récolt 's deux, trois, et même quatre fuis plus considérables que si on n'uvait pas chaulé; sans compter que les bons effuts de cette chaux se font sentir longtemps.

Q. La chaux dispense-t elle d'employer le fumier ou les autres engrais?

R. Non; et c'est une bonne pratique de funer les pièces chanlćes l'année qui suit le chaulage. Si la pièce ost en prairie, on étend le fumier en couverture après la première eoupe dn fuin.

Q. Quelles sont les piciees de terre que vous chauleriez de prefférence?

R. Les pièces de terres fortes on franches qui doivent être converties en prairies, ou en pacage.

\section{Cendres.}

Q. Combien y a-t-il d'espèces de cendres?

R. Il y a deux espèces principales de cendres: cendres vives et cendres éteintes. 


\section{Q. Quelles différences y a-t-il entre ces deux espèces} de cendres?

R. Les cendres vives sont en nature, c'est-à-dire, telles qu'elles sortes des piëles et des fourneaux; les eendres éteintes sont celles qui ont servi í lat fabrication de la potasse, du savon, ou a la lessive.

Q. Les cendres vives sont-elles toutes de la même foree?

R. Ces cendres varient dans leur force et dans leur valeur suivant les bois dont elles proviennent. Ainsi, les cendres de bois franc ont plus de foree que les cendres de bois mou; les eendres de bois, franc ou mou, orit plus de foree que les eendres de terre de savane ou de houille (eharbon de terre).

Q. Quelle est la valeur, comme engrais, des cendres vives cu éteintes?

R. Les cendres, vives ou éteintes, sont un des meilleurs engrais qu'ou puis.e employer; leur action est trèsprompte.

Q. A quelle espèce de plantes conviennent-elles surtout?

R. Elles conviennent ì toutes les plantes, surtout au blé, au seigle, à l'orge, à l'a voine, au mil et au trètle, etc.

Q Comment se fait l'éprandage de ees cendres?

R. Cet epandage se fait ì la main ou à la pelle, après la semence et sur le hersage.

Q. Quelle quantité de ecndres emploie-t-on a l'arpent?

R. On emploie de 30 à 60 minots à l'arpent, suivant la force et la valeur des cendres, et suivant l'effet qu'on veut en obtenir.

Q. Dans quels cas recommanderiez-vous, surtout, i'emploi des cendres?

R. Je le recommanderais, surtout, sur les pieces des. tinées à êtro eonverties en prairies, et jo les répandrais sur ces pièces aussitôt après les avoir enseniencées en graines de mil et de trètle, et après le hersage. 


\section{Plâtre.}

Q. A quelle espece de plantes onnvient le platre?

IR. J platre convient surtont au trèfle, a'sx pois, au tibic, aux choux, au chanvre, au lin, et au sumazin.

Qucl est l'effet du plâtre sur les círíiles, comme le ble, l'orce, le seigle, etc.?

R. Son effet sur ces plantes est ì peu près nul.

Q. Comment s'emploie le platre?

R. I t neilleure manière d'employer le plâtre est de lo répandre sur les feuilles des plantes, lorsqu'elles sont sorties de terre. On choisit de préfírence, pour cet eppondirge, le temps où les feuilles sont humides, comme le auatu après la roséc, ou après une petite pluie.

\section{Poissons, Varech.}

Q. En quelle partie du pays le poisson et le varech sont-ils employés comme engrais?

$R$. Le poisson et le varech sont employés coume engrais dans le bas du fleuve principalement, car la ils sont très-abondants.

\section{Faux sales.}

Q. Comment pouvez-vous utiliser les eaux sales comme engrais?

R. Le cultivateur soigneux doit toujours avoir, à une certaine distance de sa maison, un tas de terre ordinaire, de terre de savane, etc., sur lequel il ordonne de jeter toutes les eaux sales de la maison: eaux de plancher, eaux de cuisine, savonnures de toute espece, urines, etc.

Q. Connaissez-vous un excellent moyeu pour empêcher ces tas d'exhaler de mauvaises odeurs?

R. Oui ; ce moyen consiste à jeter, de temps en temps, quelques pelletées de terre sur ces tas. De cette manière les tas grossissent rapidement, et ne donuent pas do matuvaises odeurs. 
Q. Les tas de terre imprégnés de toutes ces caux forment-ils de bons engrais?

R. Ces tas de terre constituent d'excellents engrais qu'on ne devrait jamais négliger d'employer.

\section{Engrais chimiques.}

Q. Qu'entendez-rous par co mot engrais chimiques ?

R. On entend par ce mot certains engrais fabriqués artificiellement, qui sont destinés à remplacer les engrais ordinaires, notamment le fumier d'étable.

Q. Quel est l'engrais chimique le plus apprécié aujourd'hui ?

R. L'engrais chimique le plus apprécié est le superphoshate de chaux qui est préparé avec les os broyés et avec de l'huile de vitriol.

Q. Ces engrais ont-ils aujourd'hui une grande valeur?

R. Ces engrais ont, aujourd'hui, une très grande valeur, et en acquierent, tous les jours, une plus grande.

\section{Appropriation des semences aux terrains.}

Q. Toutes les variétés de terre conviennent-elles également bien à toutes les espèces de graines de semences?

R. Non; chaque variété de terre convient it telle ou telle espèce de semences, et ne convient que peu, ou pas du tout, à d'autres.

Q. Quelle espece de graines convient-il de semer dans les terres fortes ou franches?

R. Les graines qu'il convient de semer dans les terres fortes ou franches sont surtout: le blé, l'avoine, l'orge, certains ligumes, les navets, les carottes, les navets de Suède (choux de Siam), les chous, cte. Ces terres convicnnent surtout au trèfle et au mil, et ce sont elles que l'on doit se hâter de convertir le plus tôt possible en prairies. 
Q. Est-ce que la piéparation de la terre est la mêrne pour toutes ces especes de scmences?

R. Il s'en faut que la préparation de la terre soit la même pour toutes ces espèces do semenecs. Ain-i, pendant que l'avoine pousse bien dans une terre forte peu ameubiie, l'orge, an contraire, les navets, les navets de Sudde (choux de Siani), les carotles et les choux ne viendront bien dans ces terres que quaud elles auront été parfuitement aureublies.

Q. Donnez des cxemples.

R. Sur un seul labour, dans une prairie de terre forte, l'avoine donnera un bou rendement; pour obtenir, su contraire, une bonne récolte l'orge ou de légumes sur la même pièce, il fuut auparavant que ectte pice ait sté soumise a plusicurs labours, et à des hersarges et roulages répétís.

Q. Quclles sont les semenecs qui conviennent le mieux aux terres légères?

R. Les semences qui conviennent le micux aux terres légères sont surtout le seigrle, le sarrazin, les pois, les fêves, l'orge et les betteraves; et parmi les lígumes, les pommes de terre, les carnttes, les navets de Suede, ete.

Q. Ces eols conviennent-ils pour les prairies?

R. Ces sols ne conviennent pas pour les prairies. Ise trèfle et le mil n'y poussent qu'à force d'engrais; et ces prairies sont de courte durée.

Q. Quels sont les principanx défauts que l'on remarque en ee pays, dans l'appropriation des semences aux terrains?

R. On voit un grand nombre de cultivatenrs faire, tous les ans, des pertes considérables de temps, d'engriais et d'argent, uniquement paree qu'ils eonfient des semences a des terrains qui ne leur conviennent pas.

Q, Donnez des exemples.

R. Ainsi, on en voit yui cssaient de cultiver les navets, le blé ou l'avoine duns des terres tri, li; ères et sablenses; de l'orge, dans des terres qui ne sunt pass assez amcublies, 
etc. On en roit souvent qui s'épuisent d vouloir fairo pousser le foin sur des terres seches, tandis qu'ils ont it leur disposition de belles pieces de terres fortes ou franches qui ne demanderaient que peu de soins pour être converties en bonnes prairies de longue durée.

\section{- Ensemencements et récoltes.}

Q. Quels soins doit-on apporter dans lo choix des - graines de semence?

K. On doit apporter le plus grand soin dans le choix des graines do semenee, et les meilleures graines doivent être employées à cet objet.

Q. A quoi doit-on s'attendre quand on seme de mauvaises graines?

1R. On doit s'attendre d̀ une paurre récolte, parce que les mauvaises graines jetées sur la terre sont perdues, vu qu'elles ne peuvent germer.

Q. Quel est le défaut le plus général, en co pays, par rapport a l'ensemencement?

R. A part le peu de soin qu'on apporte dans le choix des graines de semences, on peut dire que le défaut lo plus général est qu'on sème ou trop clair ou trop fort.

Q. Quelles sont les graines que l'on doit semer fort.?

R. Les principales graines que l'on doit semer fort, sout: le blé, l'avoine, l'orge, lo seigle, le mil et le trètle.

Q. Quelles sont les graines qu'on doit semer cluir?

R. Les graines que l'on doit semer clair sont lo sarrazin, et les légumes qui doivent êtro suffisamment espacés.

Q. La quantité de graines a semer doit-elle varier suivant les qualités du sol ?

R. Oui, dans les terres bien engraissécs, la semence doit être plus claire; dans les terres peu engraissées, la scinence doit être plus forte.

Q. Quelle quantité de blé doit-on semer dans un arpent de bonne terre? 
R. Environ un minot et demi par arpent.

Q. Quelle quantité d'orge?

R. D'un minot et quart à un minot et demi.

Q. Quelle quantité d'alvoine?

R. De deux minots et demi ì trois minots par arpent.

Q. Quelle quantité de scigle?

R. D'un minot et quart a un ininot et demi par arpent.

Q. Quelle quantité de sarrazin? pent.

R. D'un demi-minot a trois-quarts de minot par ar-

Q. Quelle quantité de mil et de trèfle?

R. La quantité de mil doit être d'un quart de minot mêlé à deux livres de trêfle rougre.

Q. A quels signes reconnaît-on qu'il est temps de couper le blé, l'orge, l'avoine, le seigle et le sarrazin ?

R. Tous ces graias doivent être coupés un peu avant leur complète maturité. Ainsi, le blé est bon ì couper lorsque le grain n'étant plus en lait, est néanmoins assez tendre pour se laisser rayer par l'ongle. Dans cet état, la paille est encore verte auprès des nœuds. Tous les autres grains doivent être aussi coupês avant qu'ils soient coninplétement inûrs.

Q. Quel inconvénient y a-t-il à couper ces grains trop tard, et lorsqu'il sont tres-mîrs?

R. Il y a alors l'inconvénient qué les grains égrènent, et qu'une grande quantité de ces graines reste sur la terre, où elles sont perdues.

Q. Y a.t-il quelques précautions à prendre après la coupe du blé, du seigle et do l'orge?

R. Le blé et l'orge doivent être enorerbés et liés aussitôt après avoir étó coupés, et disposís en moyettes ou quintaux.

Q. Dites comment rous feriez ces moyettes ou quintaux.

R. On commence par mettre une gerbe debout, les épis en haut. Autour de cette première gerbe et un peu inclinées, de manictre a s'ippuyer sur elle, on dispose 
quatre antres gerbes avec les épis également en haut. Sur ces cinq gerbes ainsi disposécs, on place une grosse gerbe les épis en bas, et la moyette est prête.

Q. Quels avantages présentent ces moyeltes ou quin. $\tan x$ ?

R. Les avantages que présentent ces moyeftes ou quintaux sont considérables. Le grain ainsi disposé peut rester dehors, et par tous les temps, pendant plusieurs semaines, sans êtré nullement endounmagé. Bien pluq, disposé de cette fuçon, le grain continue à se nourrir et it unarir.

Q. Est-il aussi nécessaire de disposer l'avoine en quin$\operatorname{tau} x$

R. Il n'est pas aussi nécessaire de disposer l'avoine en quintaux, parce que l'avoine germe difficilement, et peut endurer beaucoup de mauvais temps avant de se déteriorer. Mais le blé, le seigle et l'orge germent si facilement et si promptement, qu'on doit to'jjours les mettre en quintaux, à mesure qu'on les coupe. Dans les annéees pluvieuses, le seul moyen de sauvor même l'avoine est de la mettre aussi en quintaux.

\section{Culture des légumes.}

Q. Quels sont les principaux légumes cultivés en Canada?

R. Les principaux légumes cultivés en Canada sont: la pomme de terre (patate), les navets, les ohoux, les carottes, les betteraves, etc.

Q. Quelle espice de terre convient le mieux a la culture des pommes de terre?

R. Ijespèce de terre qui convient le mieux à la culture des pomnes de terre est la terre légère, sableuse ou tufeuse.

Q. Que pensez-vous de la culture des pommes de terre dans les terres franches ou fortes?

R. Ces pommes de terres sont toujours aqueuses, et 
sujettes a pourrir promptement. Néanmoins, elles peuvent être employées avec avantage pour les engrais d'automne, ou pour la nourriture des auimaux d l'étable, jusqu'au milieu de l'hiver.

Q. Dans quelle espece de terre cultive-t-on ordinairement les navet;, les carottes, les betterares et les choux ?

R. La culture de ces légumes, servant ordinairement a préparer la terre pour la culture des grains é pour la confection des prairies, se fait, de préférence, dans la terre franche ou forte.

Q. Les divers travaux de préparation à donner a la terre poir la c'? beaucoup?

$R$. Ces travaux sont les mêmes pour tous.

Q. En quoi consistent ces travaux de préparation?

R. Ces travaux ds préparation consistent en labours profonds ct scument. - inctés, suivis do hersages et de roulages nombreuz nor bien ameublir et pulvériser la terre. Ines pièce: qui dioivent être ensemencées avec ces légumos doivent, alas si recevoir une fumure considérable.

Q. Supposant que vous vecilina srisemencer on légumes une piece de prairio useje et vieillie, quels travaux préparatoires feriez-vous?

R. La premiere chose d faire est un premior labour d'automne, aussi profond et aussi bien conditionné que possible. Le printemps suivant, cette pidce doit être ensemencée en avoine ou en blé. Le deuxième automne, nouveau labour profond; le deuxicme printemps, nouvelle semence en aroine, mais pas on blé, si la pièce a porté du bles l'année d'auparavant. Ise troisidme automne, nouveau labour; le troisieme printemps, un, deux, et même trois labours, avec autant de hersages et do roulages, suivant la force de la terre. Lorsque l'amenblissement est bien complet, la pieceo ast prête il recevoir la semence et l'engrais. 
Q. Quelle quantité de fu'nier faut-il mettre à l'arpent pour lat culture de ces iégumss? funisier.

R. Il fuut mettre is l'arpent 60 voyages ou plus de

Q. Q'ul fumier emploict-on à cet usage?

1. On emploie ordinairement le fumier de l'hiver. Si ce fumier a eommencé à pourrir avant d'être employé, cela viat mieus.

Q. Que f:ittes-vons ensuite?

R. On procède ensuite de deux manières différentes, suivant qu'ou sème la graine, ou 'gu'on plante les légumes cux-uêmes, apress qu'ils out levé dans les couches choudes.

Q- Tontes ces variétés de légunes peuvent-elles être pluntées?

R. Les choux et les navets de Suida (choux de Siam) se transplanteut; les navets oriinaires, les carottes se sement a demeure; les betterives se sim nt ou se transplintent, mais il vau mieux les semer.

Q. Quel nom donne-tron a la transplantation des légumes?

K. On donue à la transplantation des légumes le nom de repriqunge.

Q. Liensemencenent des graines de nuvets, de oarottes et de betteraves, présente-ii quelque différence ?

R. L ensemencement de ces trois espèces de graines est le nuême.

Q. Comment se fitit cet ensemencement ?

R. Il se fätic par rongss on à lie volée.

Q. Quelle est la meilleure de ces deux méthodes?

R. La meilieure ust celle qui consiste it tatre le semis par ranys; le semis ì la volée duit êtro laissé complétoment de ciôtés.

Q. Conment procédez-vous au semis par rangs?

1. Lat terre ayant éts bien ameublie et pulvérisée comme il a été dit plus hinut, reçoit un dernier labour, de manière à être toute divisée par plates-bandes étroites 
qu'on appelle billons; ces billons sont séparés par des raies. Dans chacune de ces raies on dispose le fumier également. Un trait de charrue passé dans le milieu de la longueur des deux plates-bandes ou billons qui avoisinent la raie, fait tomber la terre dans la raie et recouvre le fumier; de sorte que la où était le billon l'instant d'auparavant, se trouve une raie, et là où était une raie se troure un billon. Quand tout le fumier a óté ainsi recouvert et que les billons sont formés, on passe le rouleau, de manière a aplatir un peu leur surface. Ises billons, alors, sont prêts d recevoir la graine.

Q. Conment semez-vous la graine dans ces billons?

R. La graine dans ces billons se dépose à la mair, ou mieux a l'aide de potits instruments peu couteux qu'on appelle semoirs.

Q. Décrivez la manidre de semer d la main.

$R$. On prend une cheville pointue, ou un bois garni de plusieurs de oes chevilles placées a une distance de 10 pouces les unes des autres. Avec cette cheville, ou areo ce bois garni de plusieurs chevilles, on fait dans la plate-bande des trous ayant a peu pres un pouce de profondeur. Chacun de ces trous doit être, au moins, du la distance de 10 pouces l'un de l'autre. Dans chacun de ces trous on dépose deux ou trois graines. Ces trous sont ensuite remplis, et les graines sont enterrées avec la main, avec un râteau, ou avec une pièce de bois qu'on promene sur la plate-bande.

Q. Quel inconvénient présente cette méthode ?

R. Cette méthode est longue, fait perdre beaucoup de temps, et ne fait pas un semis régulier.

Q. Combien y a-t-il d'espèces de semoirs?

R. Il y a deux especes principales de semoirs : le semoir-brouette, ou d bras, et le semoir-à-cheval.

Q. Quel est celui qui convient lo mieux pour les usages ordinaires ? 


\section{$-41-$}

R. Pour les usages ordinaires, le semoir d bras convient lo mieux.

Q. Décrivez ce semoir d bras.

R. Ce semoir n'est autre chose qu'une petite brouette contenant une boîte ou l'on met la graine, et un petit couteau ou rayonneur qui trace sur les billons les lignes où la graine doit être déposée. La boîte à graines étant remplie, il suffit de pronsener le semoir sur les billons, et les lignes se tracent, et la graine se dépose d'elle-nêtme, suivant la quantité voulue, et aux distances convenables. Un petit mécanisıne placé dans le semoir permet d'ob. tenir tous ces résultats. Les graines sont enterrées par le semoir lui-même.

Q. Combien de temps faut-il aux diverses graines de légumes pour lever?

R. Le temps nécessaire pour les faire lever varie beaucoup, avec les différentes espèces. Ainsi, pendant que les navets sont sortis de terre 8 ou 10 jours apres leur ensemencement, les carottcs, bien souvent, n'apparaissent qu'au bout de 30 a 40 jours.

Q. Une fois que les navets sont sortis de terre, quels soins exigent-ils?

R. Aussitôt que les navets sont sortis de terre, et qu'ils ont deux feuilles un peu larges, on donne un premier sarclage pour détruire les mauvaises berbes.

Q. Comment faites-vous ces sarclages?

R. Ces sarclages se font, ou at la main, à l'aide de pioches, grattes, \&c., ou mieux, aveo un instrument peu couteux qu'on appelle houe a cheval.

Q. La houe à cheval opere-t-elle le sarclage sur la ligne même où se trouvent les navets ?

R. La houe à cheval n'opère pas le sarclage sur ces lignes; il faut le pratiquer à la main avec une espece de gratte appelée houe à la main.

Q. En mêtıe temps qu'on opère le sarolage sur ces lignes, que faut-il faire?

R. En même temps qu'on opère le sarclage sur les 
lignes, on sclaireit, c'est-ildire qu'on arrache tons les planta de navets pui se tronvent dans le inêne trou, moins un; celui qu'on liisse Init être le plus vigoureux.

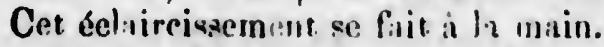

Q Qum fitut.il laire ensuite?

R. Q inze jomrs, ou trois sem uines après ce dernier sarcluge, on en fait un desxième, si le besoin s'en fit seutir, pour détrume de nouvenu les insuviisos horbes qui ont pu pnusser durant cet intervalle. Qu:nd les feuilles so'it bien develonpées et rinelles couvrent tout le terrain. ou ne sincele plus, et on laisso la végétation se fure juspra's monent ile la recoltc.

Q. Si, durant les nreniers sirclires, vons vo's apercovez que la levie dos aranes mawile en cutaing endroits, phe devez-vons fire?

R. On toit sewer d. nouvelles grines dins rea endro ts. es qui prut st fire jusque vers la tin de juillet.

Q. Quand se firit la reculte des navets?

R. Les navets que l'on divit semer, en ee pays, entre le 10 et lo 20 do juillet, sont bons ì recolter i la tin d'ortisbre.

Q. Comment se fiit l'arrachuge des navets?

R. Cet arrachiee se tait ordaarement a la main.

Q. Comment se conservent les navets?

R. On les cunierve de plusieurs manières: parfois, dans des cilves fribiches, d'autıes furs en silos, o'est-i-dire, en les recouvrant de terre.

Q. Quel est l'usilge des navetsos?

R. I.es navets sont vendus, ou nicux employes pour la nourriture des animinx.

Q. Quelles préparations fant.il faire subir ì la terre pour la culture dus wreets de Suélte (choux de Stam)?

R. Absolument les ménes yuc pour la culture des navets.

Q. Exigent-ils des soins particuliers?

R. Les soing qu'ils exigent sont absolument les mêmes que pour les narets, avec cette exception, qu'au lieu de 


\section{$-43-$}

les semer, on les transp!ante. Cette transplantation ou repiquage est le ineilieur moyen it adopter pour co légume.

Q. Conmeni se falt cette iransplantation ou repiquage?

R. Eille se fait absolunent comme pour les ehoux. On thoisit de préférenc: un lemps pluvieux ou sombre, et on met les plants d utue distanee de 11 pouces les uns des autres.

Q. En quel temps doirent étre plantés les navets do Suède?

R. Ils doivent être plantés vers la fin de mai ou le commencement de jum.

Q. Comment les soaiervet on ?

R. Un les conserve sommis les navats. Ils présentent, sur ces dernters, l'avanraze de shautec moins aisément, et de se conocrver tout l'hiver aven tacilité.

\section{Praíries.}

Q. Attachez-vous une grande importance d la préparation des prairins?

R. Oui; car le cultivatenr qui u de nombreuses et bonres praires et qui récolte beancoup de foin, est toujours un cultivateur a l'atse, at même riche.

Q. Quelles sont les pieces de terre qui conviennent le mieux pour les priciries ?

R. Les pièces qui conviennent le mieux pour les prairies sont les picces de terro for'e ou franche. Les terres légères ne peuvent être converties en prairıes qu'à force d'engrais, et eucore sontelles toujours de courte duríe.

Q. Comment vous y preadriez-vous pour faire une belle et bonne prairie?

R. La premiche chose il fitire est de choisir une piéce de terre convenuble, cost-a-dire une piece de terre forle ou franche, et ensuite de la bien égoutter, en pristiquant 
ou creusant des fossés, si nicux l'on n'aime avoir recours au druinuge. Ceci étant fuit, les moyens a prendre sont de deux espèces; suivant qu'on veuille préparer la pièce a l'uide seule des labours, ou qu'on veuslle la préparer à l'aide de la culture des légrinzes.

Q. Décrivez la meiliode à employer avec l'aide sculc des labours.

R. Supposant que la pièce que je veux traiter soit une prairie vicillı., courerte de mousse et usée, la première chose à faire est un bon labour d'autonace. Le printemps suivan, semence en avoine oli en bit, avers mil et trefle, ut vigoureux hersage.

Q. Que pensez-vous de cette methode?

R. Cette incithode est do toutes la plus économique, vu qu'elle cxige peu de travail et nul engrais. Dans les terres trd̀s-bonnes et fertiles par elles-mênes, cette méthodo suffit pour façonner d'ussez bonnes prairies qui durent pendant einq ou six ans. C'est la méthode que peuvent employer les cultivateurs très-paiveres qui n'ont que trèspeu ou point d'engrais, et n'ont pas les moyens de s'en procurer. Le grand inconvénient de cette méthudi est que les mauvaises herbes ue tardent pas a pousser.

Q. Pouvez-vous, ù peu de frais, améliorer cetteméthode?

R. On peut, sans beaucoup de frais, améliorer cetfe méthode, en ayant recours à l'une ou à l'autre des deux especes de chaulages qui onr cié décrits ci-devant. Avec ces chaulages, lis prairie rendra beancoup plus, et beatlcoup plus longtempip.

Q. Supposons que le cultivatcur puisse disposer de trente à quarante voyages de fumicr, peut.il, en suivant cette méthode, utiliser cc funier pour iugrmanter l: rendement de sa prairie et sa durée?

R. Oui, et c: qu'il a de miçux à fairc alors, est de laisser pourrir es: funier avec les prévationo données plus haut. Aussitôt apris que le grain aura été enlevé. il etendra ce fumice bicn syalement sur sa prairie; et bientôt il verra lo tritle et le mil reprendre une vigueur 
e seulc

er soit

la pre.

C. Lo

, averg

que, vu

terres

éthode

durent

uvent

e très-

ic s'en

de est

hode?

cette

deux

$\Lambda$ vec

beall-

er de

ivant

: lo:

st de

nées

levé,

; et

leur 
extraordinaire. S'il ne pouvait faire cette couverturo la première année, il pourrait la faire la seconde ou la troisième, si la terre n'était pas trop compacte.

Q. Le fumier peut-il être êtendu en couverture sur toutes les espèces de terres?

$R$. Le fumier en couverture n'agit bien que sur les terres ameublies; sur les terres trop compactes, le jus du fumier ne peut pas pénétrer dans la terre et s'écoule dans les raies, les rigoles et les fossés.

Q. Comment préparez-vous uno prairie a l'aide de la culture des légumes?

R. Pour préparer une prairie à l'aide dez légumes, on procède d'abord comme il a été dit plus haut d propos de leur culture. Le printemps qui suit la récolte, on laboure, on ensemence en orge, en aroine, ou en ble, avec graine de mil et trétle. Une prairie faite do cetto manière pousse avec une vigueur extraordinaire, donne de trois à quatre cents bottes à l'arpent, et dure sept, huit et dix ans.

Q. Quels inconvenients mencontre t-on dans la próparation des proiries, surtout lorsque la terre n'est pas préparée d'avance par la culture des légumes?

R. Les inconvinients que l'on rencontre sont, d'abord, la difficulté de bien conditionner le labour, le hersage et le roulaue, via que ees opérations, ayant lieu de bonne heure le printemps, ou tard l'automne, l'humidité trop grande du sol s'oppose a ce que ees travaux soient bion ian, cisurte, par la culture des légumes, la terre est bien mieux ameublie, et surtout mieux débarrassée des mauvaises herbes de toute espice.

Q. Connaissez-vous un moyen économiquo qui permet de remódier id ces ituconrénients "?

R. Un moyen qui permet de remédier à ces inconvenients et qui, en mône teınps, nous met en état de farre de belles et bonues prairies, est la inéthodo dito de juchère.

Q. Lecrivez cette méthode. 
R. Supposant qu'il s'agisse de traiter en juchère une prairie déja vicille et usée, on commence par en tirer deux récoltes d'avolne, a l'aide de labours d'automne, comme il a été dit plus haut. Aprés la deuxième réenlte d'avoine, nouveau labour jacionne; et le troisiène printemps, eette piece ust hersés, mais pas ensemencée. Durant la morte stceson de l'eté, au mois d'août, on liboure de nodvas cette terre. 15 la herse et on ia roule.

Q. Q'el est l'ubjet de cous ces labours, hersages et roulinges?

R. Liobjet de ons ees iabours. hersages et roulages est $1^{0}$ de bien anneubir la cerre; $2^{\circ}$ do ditruire toutes les mauvaises plantes qui, étant déchassées a mesture qu'elles poussent, se trouvent exposees a l'aution du solew qui les détruit?

Q. Que f'eriez-vous ensuite?

R. Après le dernier iabour, vers le milien ou la fin d'ilat on ensemence cette piece en mil et triffe, a raison de $\&$ de minot de mil et 2 livres de trifte, bien mêlés ensemble, à l'arpent. La graine est enterrée par un hersage. Si, durant deux ou trois des labours qui ont precéde, le culrivateur a pu faire un chisulage, on s'il peut répandre sur ce terrain, en cuuperture, innmititement sur la semence, 20 ou 30 tombereaux de fumier pourri, il est sûr de fiıre une prairie de preusice ordre, et qui durera longtemps.

Q. Quelle est votre opinion sur la valeur de toutes ces méthodes?

R. La première méthode avee un seul labour, sans chaulage et sans couverture, et celle par la j r hiere sans chaulage et sans eouverture, ne eonviennent. qu'aux cultivateurs tellement pauvres qu'ils ne penrent pas disposer de quelques voyages de funier, ou do ruclipues piastres pour l'achat de einq ou six barrigues de chaux; et cey cas sont rares. La promière méthode avec chauJage ou fumier en converture, et la mothsle par la juchère aveo chaulage, ou fumier en couverture, sont 
peut-être les meilleures ì employor pour un tround nombre de cultivatenrs qui commencent à amelicrer, et qui n'ont pits beancoup d'engrais a leur dispigition. Mais aussitôt que le temps et les innyers do tíaire la culture des légumes le perinettront, et dés lors que la connaissance les avantages qur: présente cette culturi des légumes sera suftisument répanduc, le cultivateur devra se láter d'ensemencer en légumes all moins uis arpent par anrée.

Q. Quels soins réclament les prairies une fois qu'elles sont fuites?

R. Jse prenier soin ì donner aux prairies, une fois quelles sont fuites, est de bien roir a co que l'égouttement soit toujours parfait. Pour cela, il faut bien veiller, tous les antomnes, à vider les raies, les rigoles et les l'ussés, et voir à ce que rien ne s'oppose au libre écoulemont de l'eau.

Q. Quel est l'cffet du séjour de l'eau sur les prairies?

R. Cet elfet est des plus désastreux, a cause des grolies.

(?. En quel temps convient-il de couper le foin?

k. Le meilleur temps pour couper le foin est lorsque les Heurs du mil commencent a tomber. Ceux qui ont beaucoup do toin i faire feront nieux de eommencer un peu plus töt.

Q. Wuels sont les principaux défauts que l'on romarque, en Canud., dans l'entretien des prairies?

K. Ces deliats sont nombreux: $1^{0}$ dans bien des arrondissencents, les prairies sont trop vieilles, et n'ont has str levés lepuis 15,25 , et même 40 ans; $2^{\circ}$ ces prinines, couvertes de mousse et de mauvaises herbes, ur: rapporterst plus que quelques royages de mauvais foir; "3" on ne veille pas a l'entretien des raies, des riggles el destussés, de sorte que l'eal séjourne sur ecs prauries et detruit les racines du foin; $4^{\circ}$ on ne procède i la coupe du foin que quand il est tris-mur, de sorte 
que ce foin, ayant perdu toute sa grains, ne raut guère mieux que la pail'c.

\section{Pacages.}

Q. Est-il important d'avair de bons pacuges?

R. Il est très-inuportant d'avoir de kons pacrges, parce qu'il ne suffit pas de bien entretenir ies animaux pendant l'hiver, il faut encore les bien nourrir durant I'été.

Q. Comment doit-on préparer les pacrages?

R. On doit prépirer les pacagrs conme on prépars les prairies; les meillears pacuges sont les piices de terre qui ont été en prairies pendint quelques années.

Q. Quels sont les défiuts les plus remarquables que l'on observe dans les pacriges de ee pays?

R. Jes pacages, en ce pays, sont génúralement paurres; cela depend de ce qu'on ne se donne pas la peine d'ensemencer les pièces en mil et triffe, et que nos pucrages ne sont généralement que les chaumes des années pré. cédentes.

Q. Quel avantage y aurait.il à cnsemencer les pacages en mil et trètle?

R. Ces avantages consisteraient, surtout, en ce que l'herbe étant bien plus fournic, on ne scrait pas obligé de laisser en paeage d'aussi grandes étendues de terre qu'on le fait aujourd'hui; et, ensuite, les aninaux, ayant une nourriture plus abondante, donneruient plus de revenu.

\section{Animaux.}

Q. Qucls sont les principaux animaux que l'on eldeve en ce pays?

R. Les principaux animaux qu'on élère sont les chevaux, les vaches, les pores et les moutons. 


\section{Vachés.}

Q. Quelle quantité de lait doit donner une bonne vache?

R. Une bonne rache doit donner, au moins, cinq ou six pots de lait. Ce lait doit être blanc et fournir une bonne quantité de crême.

Q. Quelle quantité de lait donnent, en moyenne, les meilleures vaches?

R. Les meilleures vachies dnnnent de huit a neuf pots de lait. Quelques-unes donnent jusqu'id dix et douze pots; mils ese vaches sont rares.

Q. ILes vaches qui donnent le plus de lait sont-elles toujours les meilleures?

R. Les vaches qui donnent le plus de lait no sont pas toujours les meilleures; car il arrive, quelquefois, que ce lait très-abomiant est paure, et donne peu do crême.

Q. Comment juge-t-on ordinairement do la qualite du lait.?

R. On en juge ordinairement par la couleur: le lait riche est blanc, le lait paure est bleu.

Q. Quel'es sont les races de vaches étrangères qui conviennent le micux à ce pays?

R. An dire des connaisseurs, la race etrangere qui convient le nieux is notre climat, est la race $A$ yrshire.

Q. Que pensez-vous de notre race canadienne?

Q. On trouve d'excellentes vaches de race canadienne, lorsqu'on se doune lit peine de les bien choisir. Les vaches moitié ayrshire et moitié cunadienne's sont excollentus.

Q. Quelz soins particuliers exigent les vaches?

R. Durant la saison de l'été, elles dnivent avoir de boins pacages; durant l'hiver, elles doivent avore une bonne nourriture, et être tenues proprement.

Q. Comment les vaches doivent-elles être nourries a l'stable?

R. Les vilches doivent être nourries al l'étable areo de 
bon foin or de bonne paille, et avoir un repas de legumes, au moins, on une boilte par jour.

Q. Quels sont les logumes qui conviennent le mieux i la nourriture des vaches?

R. Les légumes qui vonviennent le mieux aux vaches wont les botteraves, les carottes, les navets de Suedo (ohoux de siam), les navets, les pommes de terre, eto.

Q. Qnelle précantion faut-il prendre avant de donner ces logumes aux vaches ?

R. Il faut avoir soin de les couper par tranches ou par petits morceaux; si l'on ne prend cette précaution, il arrive quelquefois que les vaches avalent de trop gros morceaux et s'tlouffent.

Q. Comment coupe-t-on ces légumes?

R. On les coupe à l'aide de couteanx, de tranches, ou mieux aveo un instrument particulier appeló coupe. racines.

Q. Qu'entendez-vous par ces mots: tenir lee vaches proprement?

R. J'entends que les vaches doirent être écurées tous les jours, qu'elles doivent avoir assez d'espace dans l'étable pour y reopirer un air pur, et que, de temps a autre, on doit les étriller.

Q. Croycz-rous qu'il soit bien important de downer un repas de légumes aur vaches par jour?

R. Cela est très important. Ce repas de légumes est le plus sar mojen de tenir les vaches en bonne santé; sans compter qu'areo ces légumes, les vaches donnent beaucoup plus de lait, et le gasdent bien plus longtemps?

\section{Porcs.}

\footnotetext{
Q. Que pensez-vous de la valeur de nos porcs canadiens?

R. La race de nos porcs canadiens est tout a fait inférieure, et somplétement dégónérée. Ces pores mangent beaucoup, et engraissent lentement.
} 
gut

eux

hes

edo

ner

ou

on,

ros

ou

pe.

hes

us

as

d

er

st

t' 
(1) 
Q. Quelles sont les prinoipales races otrangores intro duites en ce pays?

R. Les principales races étrangdres cont celles des Suffolk, Berkshire, Yorkshire, eto.

Q. Quels avantages présentent ces porcs de races étrangeres sur nos pores canadiens ?

$R$. Ils engraissent beaucoup plus facilement, beaurooup plus vite, tout en mangeant beaucoup moins.

Q. Quelle est la meilleure saison pour l'engraissement des poros?

R. La meilleure saison est l'été, et lo commencement de l'automne; d'abord, parce que ces animaux engrairsent beancoup mieux a la chalour qu'au froid; ensuite, parce que, durant l'été, une bonne partie de l'engraissement peut se faire avec le lait des vaches.

\section{Moutons.}

\section{Q. Que pensez-vous de notro race de moutons cana- diens?}

R. Notre race de moutons, comme celle de nos poros, est tout a fait dégénérée, et doit être renouvelée.

Q. Quelles sont les races étrangères qui conviennent lo mieux a ce pays?

R. Après de nombreux essais, les connaisseurs paraissent s'accorder à dire que ce sont les races onnnues sous les noms de: Cotsioold, South-down ou un mélange des deux.

Q. Quels soins exigent les moutons à la bergeris?

R. Les moutons doivent être tenus fraîchenient. $L_{\mathbf{a}}$ porte de leur bergerie doit être laissíe presque toujours ouverte dans le cours de lhiver, excepté dans les froids très-rigoureux; ct une bonne précaution est d'avoir un enclos en avant de la bergerie où ils puissent sortir en plein air quand bon leur sensble.

Q. Quelle nourriture oonvient le mieux sux moutons

R. La nourriture qui convient le mieux aux moulons est le treffe, le foin, le pesa : et si on peut leur donner 
un repas de légumes chaque jour, ils donneront plas de laine, et auront beaucoup plus de chance de se tenir en bonne santé.

Q. Quand le cultivatour doit-il songer à faire l'achat d'animaux améliorés?

$\dot{R}$. Ise cultivateur ne doit songer \& faire l'achat d'animaux améliorés que lorsqu'il est sûr que les produits de sa terre suffiront a les bien nourrir et a les entretenir. convenablement. Ainsi, il ne doit songer ì élever ou a acheter des vaches améliorées que lorsque ses prairies donneront un assez fort rendemont pour lui permoltre de donner a ses vaches une quantité suffisante de bon foin durant l'hiver, et lorsqu'il aura d'assez bons pacages pour qu'elles puissent $y$ trouver une abondante nourriture durant l'été.

Q. Que deviennent les animaux amćliorés, lorsqu'ils ne sont pas bien nourris et entretenus eonvenablement? valeur

R. Ils dégénerent promptement, et perdent toute lisur

\section{Instruments agricoles.}

Q. Enumérez quelques-uns des instruments agricoles nouveaux ou perfectionnés qui sont peu coûteux, et qu'un cultivateur intelligent doit avoir.

R. Ces instruments sont: les fuuchcuses, le javelier, le ratexu d-cheval, et un instrunont bien commode, mais peu connu qui scrt à engerber, et portc le noin d'engerbeur. Ceux qui cultivent les legumes (navets, navets do suède, etc.), doivent se munir d'un semoir it bras, d'une houe à cheval. Pour l'enlèvement des mavaises herbes (chiendent, marguerites; etc), l'extivpateur ou cultivateur est un instrument bien prícicux. Cet instru. ment sert aussi a ameublir le sol profondément.

\section{Rotations.}

Q. En quoi consiste la rotation? 
R. La rotation consiste ì faire succeder les dirersea plantes les unes aux autres sur une même pièce do terre, de manière à retirer du sol la plus grande consse de produits avee le moins de dépenses possible.

Q. Qu'entendez-vous par ces mots: fairc succeder les diverses planies les unes anx autres?

R. Ces mots signifient, qu'ì part de très rareo e.xcep. tions, on ne doit pas semer la mêtue graine, ou des grinines du même genre, dẹx oil plusicurs amnses de suite sur la même pièce de terre.

Q. Quel inconrénicnt y il-t-il à semer les mèmes graines deux ou plusieurs années de suite sur la mine pièce?

R. L'inconvénient est que cette graine ne trouve plus la nourriture dont elle a hesoin.

Q. Expliqnez pourquoi la graine ne trouve plus la nourriture dont elle a besoin.

R. Il est reconnu que chaque espèce de graines en. lève à la terre une certaine proportion des matières nutritives que celle-ci renferme; il est reconnu encore que ces matières nutritives sont de diverses espèces, et qu'elles ne sont pas enlevées également et dans les mêmes proportions par les diverses plantes. Ainsi, par excmple, tandisque certaines plantes enlèvent au sol beaucoup de chaux et peu de potasse, d'autres, an contraire, lui enlèvent beaucoup de potasse et peu de chaux, etc. D'après cela, il est évident que si l'on sème deux, ou plusieurs années de suite, la même graine sur le même terrain, ectte graine finira par ne plus trouver en assez grande proportion la nourriture dont elle a le plus besoin.

Q. Donnez des exemples.

R. Le blé ne doit pass être semé deux années de suite sur la même pièce de terre, non plus que l'orge, le seigle, les légumes, etc.

Q. Les rotations sont-elles bien nombreuses?

R. Les rotations sont très-nombreuses et varient suivant les qualités du sol et les divers pays. 
Q. Quelle est la rotation qui convient le mieux, dans les terrey fortes ou franches, en ce pays?

R. Lia rotation qui convient le mieux est celle qui permet de récolter le plus de foin, et d'obtenir les meilleurs pacages, afin de pouvoir nourrir et entretonir le plus grand nombres d'animaux.

Q. Donnez des exemples.

R. Ces exeinples ont été donnés ci-devant, (page 43) a propos de la préparation des prairies : $1^{\circ}$ par les labours seuls; $2^{\circ}$ par la culture des légumes; $3^{\circ}$ par la jachere.

Q. Quels défauts remarquez-vous dans les rotations suivies en ce pays?

R. Le principial défaut à observer est que dans maints endroits il n'y a pas de rotations du tout. On oultive au hasard, ct sans intelligence; on a une pièce à pois sur laqquelle on sème toujours des pois; une pièce à blé qu'on ensemence toujours en ble, et ainsi de suite.

Q. Quelle est la conséquence de cette pratique?

K. La conséquence de cette pratique est qu'on ruine la terrc, et qu'en la ruinant, on se ruine soi-même.

\section{Système de culture.} ter?

Q. Quel système le cultivateur canadien doit-il adop-

R. Le cultivateur doit adopter pour système de convertir lc plus promptement possible, et aussi parfaitement que le temps ct ses moyens le lui permettent, la plus grande étendue possible de sa terre en prairies.

Q. Pourquoi le cultivateur doit-il chercher a convertir la plus grande étendue possible de sa terre en prairies ?

R. Pour plusicurs raisons. D'abord, les terres, en ce pays, ont une grande étendue et la tuain-d'oevre vient rare; ensuite la saison de l'étét est fort courte, de sorte qu'on doit chercher, avant tout, à diminuer la somme des travaux ì cxécuter.

Q. Comment la culture du foin rous permet-el. de diminuer la somme des travaux? 


\section{$-55-$}

R. Parceque, lcs prairies, une fois qu'elles sont bien faites, n'ont pas besoin d'être labourées l'automue, ni ensemencées le printemps, zxcepté lorsqu'elles ont vieilli, ct que le temps est venu de les relever. Elles n'ezigent d'autres travaux que le fauchage et le rentrage du foin.

Q. Quel autre avantage présente ce système?

1R. Ce système permet de récolter beaucoup de foin. Or, avec beaucoup de foin, on peut entretenir un grand nombre d'animaux en bon ordre. Ces animaux donnent beaucoup de produits qui rapportent de grands profits, et, en outre, une grande quantité d'engrais. L'engrais est tellement la base de toute bonne agriculture qu'on dit généralement, et avec raison, quo le fumier est le capital du cultivateur. 


\section{TABLE.}

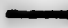

Pageo.

Nutrition des Plantes....................... 5

Especes de terres.......................... 6

Amendements ........................... 7

Travallx de la terre....................... 9

Lallour ................................. 9

Hersage............................... 13

Roulage $\ldots \ldots \ldots \ldots \ldots \ldots \ldots \ldots \ldots \ldots \ldots \ldots \ldots 15$

Egontlement .......................... 16

Engrais et Furiers ....................... 19

Funuer d'étables. ........................... 20

Trastement des fumierz...................... 22

Diverses especes de funiers................... 24

Engrals verls........................... 26

Chaux et Chaulage.......................... 27

Cendres ................................. 30

Plàtre .................................. 32

Poiseons, varech......................... 32

Euxx salea ............................. 32

Engrais chimiques......................... 33

Appropriation des semences aux terrains.......... 33

Ensemencement et récoltes.................. 35

Culture des lègumes $\ldots \ldots \ldots \ldots \ldots \ldots \ldots \ldots \ldots . \ldots 37$

Prairies............................... 4.3

Pacages.............................. 48

\&umalix......................... 48

Vaches .............................. 49

Porcs.............................. 60

Moutons............................ 51

Instruments agricoles.................... 62

Rotations............................... 52

Systeme de culture....................... 64 



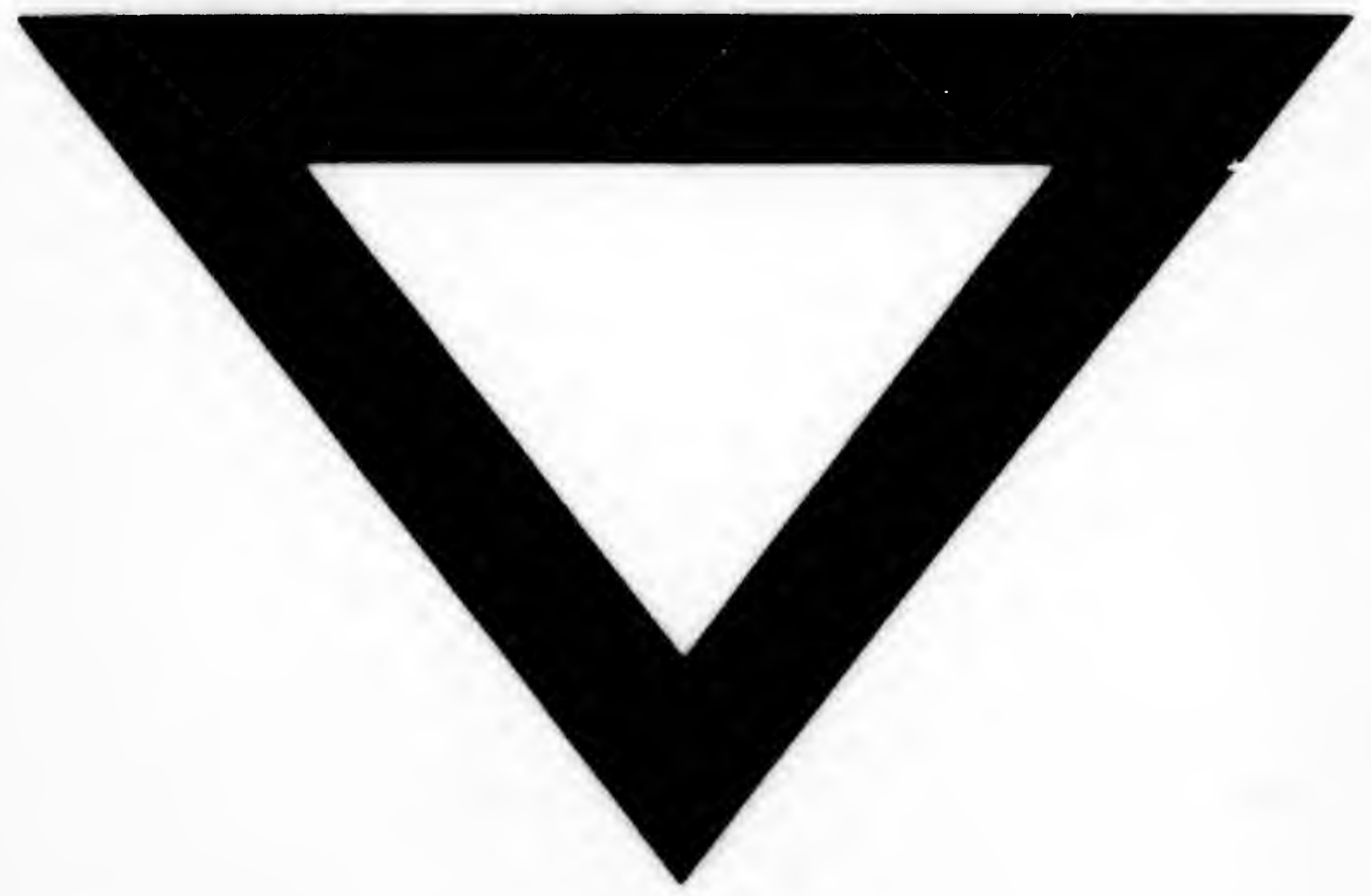

